\title{
Lyapunov-Based Model Predictive Control of Nonlinear Systems Subject to Data Losses
}

\author{
David Muñoz de la Peña and Panagiotis D. Christofides
}

\begin{abstract}
In this work, we focus on model predictive control of nonlinear systems subject to data losses. The motivation for considering this problem is provided by wireless networked control systems and control of nonlinear systems under asynchronous measurement sampling. In order to regulate the state of the system towards an equilibrium point while minimizing a given performance index, we propose a Lyapunov-based model predictive controller which is designed taking data losses explicitly into account, both in the optimization problem formulation and in the controller implementation. The proposed controller allows for an explicit characterization of the stability region and guarantees that this region is an invariant set for the closed-loop system under data losses, if the maximum time in which the loop is open is shorter than a given constant that depends on the parameters of the system and the Lyapunov-based controller that is used to formulate the optimization problem. The theoretical results are demonstrated through a chemical process example.
\end{abstract}

Index Terms-Fault-tolerant control systems, networked control systems (NCS), predictive control for nonlinear systems, process control applications.

\section{INTRODUCTION}

$\mathbf{M}$ OST control systems are designed under the assumption of flawless communication at the sensor-controller and controller-actuator links and continuous or synchronous measurement sampling. These assumptions hold in most applications where point-to-point communication links are used and measurements of velocity, position or temperature are fed into the control system. However, nowadays there is an increasing number of industrial processes controlled via a shared communication network, see for example [1]-[3]. Control systems which operate over a communication network (wired or wireless) are known as networked control systems (NCS) and can substantially improve the efficiency, flexibility, robustness and fault-tolerance of an industrial control system as well as reduce the installation, reconfiguration and maintenance costs. In addition to dealing with complex process dynamics (e.g., nonlinearities and uncertainty) and enforcing certain optimality properties in the closed-loop system, NCS have to account for the dynamics introduced by the communication network. In general, network dynamics are

Manuscript received January 10, 2007; revised February 15, 2008. Current version published October 8, 2008. This work was supp orted by the National Science Foundation (NSF), CTS-0529295 and MEC, DPI2007-66718-C04-01. Recommended by Associate Editor M. V. Kothare.

D. Muñoz de la Peña was with the Department of Chemical and Biomolecular Engineering, University of California, Los Angeles, CA 90095 USA. He is now with the Departmento de Ingeniería de Sistemas y Automática, Universidad de Sevilla, Sevilla 41004, Spain (e-mail: davidmps@ cartuja.us.es).

P. D. Christofides is with the Department of Chemical and Biomolecular Engineering and the Department of Electrical Engineering, University of California, Los Angeles, CA 90095 USA (e-mail: pdc@ seas.ucla.edu).

Digital Object Identifier 10.1109/TAC.2008.929401

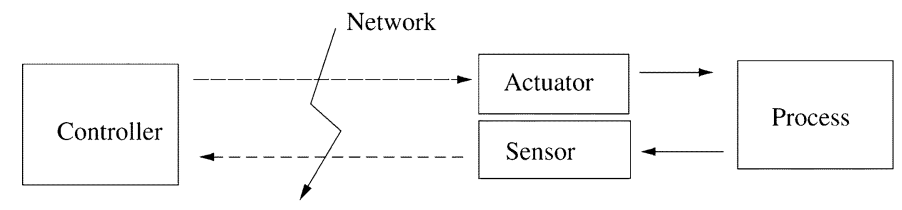

Fig. 1. Networked control system subject to data losses.

modeled as time-varying delays, data quantization or data losses. In this paper, we focus on the design and analysis of NCS for nonlinear systems subject to data losses. Fig. 1 shows a schematic of a NCS, where data can be lost at the sensor-controller and controller-actuator links. In both cases feedback is lost, and the actuator must operate on its own, usually setting the control input to zero or to the last implemented value. Although data losses may affect any NCS, the networked control formulation of Fig. 1 (which is made precise mathematically in Section II below) is of particular interest for wireless networks, where, in general, data losses due to interference is the main dynamic introduced by the network in the closed-loop system. Wireless networks have received a lot of attention lately, see [4]-[7] and the references therein, since they play a prominent role in several areas of interest, like sensor networks [8], [9], multi-agent systems [10], [11] and chemical process systems [12] where a wide range of wireless actuators and sensors is nowadays available from vendors. In addition to NCS, the results presented in this paper are also relevant for processes in which the sensor data are received by the control system in an asynchronous manner due to the difficulties of measuring certain process variables such as the concentration of different species. In this case, the samplings are not received at fixed time instants which are roughly equally distributed. Both NCS subject to data losses and systems with asynchronous measurements (see for example [13]) can be modeled as asynchronous systems of the type considered in this work; that is, systems with strongly coupled continuous and discrete variables in which the continuous variables obey continuous time ordinary differential equations and the evolution of the discrete variables is randomly determined [14].

Although there are many works in the literature focusing on the analysis and design of NCS, there are few results focused on NCS subject to data losses. In [15], stability and disturbance attenuation issues for a class of linear NCS subject to data losses modeled as a discrete-time switched linear system with arbitrary switching was studied. In [16] (see also [17]-[19]) optimal control of linear time-invariant systems over unreliable communication links under different communication protocols (with and without acknowledgement of successful communication) was investigated and sufficient conditions for the existence of stabilizing control laws were derived. In [14], the stability prop- 
erties of a class of NCS modeled as linear asynchronous systems was studied. NCS in which the plant is modeled by a nonlinear system have received less attention. Limited access systems where each unit must compete with the others for access to the network have been studied in [20]-[23] within a sampled-data system framework. In these works, practical stability of the system is guaranteed if the maximum time for which access to the network is not available is smaller than a given constant denoted as the maximum allowable transmission interval (MATI). In [7], general nonlinear NCS with disturbances are studied and a deterministic treatment of dropouts is presented. Other recent results deal with the stability of continuous nonlinear systems under Lyapunov-based control subject to data losses [12]. A common theme of the above-mentioned works is that the controller is designed without taking into account the network dynamics and subsequently, the robustness of the closed-loop system in the presence of the network dynamics is studied.

In another recent line of work, Antsaklis and co-workers [24], [25] have proposed a strategy based on using an estimate of the state computed via the nominal model of the plant to decide the control input over the period of time in which feedback is lost between consecutively received measurements. In [24], [25] this framework was applied to optimize the bandwidth needed by a networked control system modeled as a sampled-data linear system with variable sampling rate. Other relevant works related to this approach include [26], [27], where the design of a linear output-feedback controller to stabilize a linear NCS in the presence of delays, sampling and data losses was addressed, and [28], [29] where different control schemes were applied to a magnetic levitation test-bed controlled through a network.

In the present work, we adopt the model-based control approach to dealing with data losses and asynchronous measurement sampling in the control system and propose a Lyapunov-based model predictive controller for a broad class of nonlinear uncertain systems that is designed taking into account both disturbances and data losses. Model predictive control (MPC) is a popular control strategy based on using a model to predict at each sampling time, the future evolution of the system from the current state along a given prediction horizon. Using these predictions, the input trajectory that minimizes a given performance index is computed solving a suitable optimization problem. To obtain finite dimensional optimization problems, MPC optimizes over the family of piecewise constant trajectories with a fixed sampling time and a fixed prediction horizon (i.e., a fixed length). Once the optimization problem is solved, only the first input is implemented, discarding the rest of the trajectory and repeating the optimization the next sampling step (the so called "receding horizon scheme"). See for example [30], [31] for a review of results in this area. In our opinion, the MPC framework is particularly appropriate for controlling systems subject to data losses because the actuator can profit from the predicted evolution of the system, to update the input when feedback is lost, instead of setting the input to a fixed value (normally to zero or to the last computed input).

In order to guarantee robust stability of the closed-loop system, MPC controllers must include a set of stability constraints. Different schemes can be found in the literature, see
[32] for a review on MPC stability results. We consider a Lyapunov-based model predictive control (LMPC) scheme [33]-[35] (see also [36], [37]) based on uniting receding horizon control with control Lyapunov functions, because it allows for an explicit characterization of the stability region and a reduced complexity optimization problem. Other approaches like min-max formulations [38]-[42] do not have a priori closed-loop stability regions and in general have a higher computational burden. LMPC has been applied with success to constrained non-linear systems, switched systems and in fault-tolerant control schemes [33]-[35].

The main idea of LMPC is to formulate appropriate constraints in the predictive controller's optimization problem based on an existing Lyapunov-based controller, in such a way that the MPC controller inherits the robustness and stability properties of the Lyapunov-based controller. However, previous results on LMPC [33]-[35] are based on the assumption of flawless communications. When data losses are taken into account, these schemes are not guaranteed to maintain the desired closed-loop stability properties.

In the present work, we modify the previous LMPC controllers to take into account data losses, both in the optimization problem formulation and in the controller implementation. In the LMPC scheme proposed in the present work, when data is lost, instead of setting the control actuator output to zero or to the last available value, the actuator implements the last optimal input trajectory evaluated by the controller (this requires that the actuator must store in memory the last optimal input trajectory received). The proposed LMPC scheme inherits the stability and robustness properties in the presence of uncertainty and data losses of the Lyapunov-based controller, while taking into account optimality issues. Specifically, the proposed LMPC scheme allows for an explicit characterization of the stability region, guarantees practical stability in the absence of data losses, and guarantees that the stability region is an invariant set for the closed-loop system under data losses if the maximum time in which the loop is open is shorter than a given constant that depends on the parameters of the system and the Lyapunov-based controller that is used to formulate the optimization problem.

This paper is organized as follows. In Section II the class of nonlinear systems considered in this work is introduced as well as Lyapunov-based model predictive controllers and a general class of Lyapunov-based controllers. In Section III, some properties of the Lyapunov-based controllers are established. In Section IV, the proposed LMPC scheme is introduced along with its stability and robustness properties. In Section V, the theoretical results are demonstrated through a chemical reactor example. In Section VI, we summarize our results.

\section{PRELIMINARIES}

\section{A. Notation}

The operator $|\cdot|$ denotes Euclidean norm of a vector. Class $\mathcal{K}$ functions, $\alpha_{i}(s)$, are strictly increasing continuous functions of their argument and satisfy $\alpha_{i}(0)=0$. We use $\Omega_{r}$ to denote the set $\Omega_{r}:=\left\{x \in R^{n_{x}} \mid V(x) \leq r\right\}$. The operator "/" denotes set substraction, i.e., $A / B:=\left\{x \in R^{n_{x}} \mid x \in A, x \notin B\right\}$. 


\section{B. System Definition}

In this work, we assume that the process in Fig. 1 is modeled by a nonlinear system subject to disturbances with the following state-space description

$$
\dot{x}(t)=f(x(t), u(t), w(t))
$$

where $x(t) \in R^{n_{x}}$ denotes the vector of state variables, $u(t) \in$ $R^{n_{u}}$ denotes the vector of input variables, $w(t) \in R^{n_{w}}$ denotes the vector of disturbance variables, and $f$ is locally Lipschitz on $R^{n_{x}} \times R^{n_{u}} \times R^{n_{w}}$. The disturbance vector is bounded, i.e., $w(t) \in W$ where

$$
W:=\left\{w \in R^{n_{w}} \text { s.t. }|w| \leq \theta, \theta>0\right\} .
$$

Note that uncertainty must be introduced in the model in order to consider the deviation of the real state of the system from the one estimated using the model.

\section{Lyapunov-Based MPC}

LMPC is based on uniting receding horizon control with control Lyapunov functions and computes the manipulated input trajectory solving a finite horizon constrained optimal control problem. While there are several LMPC schemes that have been proposed in the literature (see for example [36], [37]), in this work we design the proposed LMPC using the results developed in [33]-[35]. In order to define a finite-dimensional optimization problem, the manipulated input trajectory (i.e., the free variable of the LMPC optimization problem) is constrained to belong to the family of piece-wise constant functions $S(\Delta)$ with sampling period $\Delta$ and length equal to the prediction horizon. This means that although system (1) is defined in continuous time, the control input is a piece-wise constant function of time and the resulting closed-loop system is a nonlinear sampled-data system with sampling time $\Delta$. As mentioned in the introduction, LMPC is characterized by a set of constraints based on an existing Lyapunov-based controller. This set of constraints guarantees that the LMPC inherits the same stability and robustness properties of the Lyapunov-based controller when it is applied in a sample-and-hold fashion.

To proceed with the presentation of the LMPC, we need to make certain assumptions. First, we assume that the nominal closed-loop system (system (1) with $w(t) \equiv 0$ for all $t$ ) has an asymptotically stable equilibrium at the origin $x=0$ for a given feedback control $h: R^{n_{x}} \rightarrow R^{n_{u}}$ which satisfies $h(0)=0$ (this assumption is equivalent to the existence of a control Lyapunov function (CLF) for the system $\dot{x}=f(x, u, 0)$ ). This feedback law will be used in the design of the LMPC controller. Using converse Lyapunov theorems (see [43], [44]), this assumption implies that there exist functions $\alpha_{i}(\cdot), i=1,2,3,4$ of class $\mathcal{K}$ and a Lyapunov function $V$ for the nominal closed-loop system (system (1) with $u(t)=h(x(t))$ and $w(t) \equiv 0$ ) which is continuous and bounded in $R^{n_{x}}$, that satisfy the following inequalities

$$
\begin{aligned}
& \alpha_{1}(|x|) \leq V(x) \leq \alpha_{2}(|x|) \\
& \frac{\partial V}{\partial x} f(x, h(x), 0) \leq-\alpha_{3}(|x|) \\
& \left|\frac{\partial V}{\partial x}\right| \leq \alpha_{4}(|x|)
\end{aligned}
$$

for all $x \in D \subseteq R^{n_{x}}$ where $D$ is an open neighborhood of the origin. We denote the region $\Omega_{\rho} \subseteq D$ as the stability region of the closed-loop system under controller $h(x)$. Note that explicit stabilizing control laws that provide explicitly-defined regions of attraction for the closed-loop system have been developed using Lyapunov techniques for specific classes of nonlinear systems, particularly input-affine nonlinear systems; the reader may refer to [45], [46] for results in this area. In Section V, a method such as the one presented in [47] is used for the design of $h(x)$. In the remainder, we will refer to the controller $h(x)$ as the Lyapunov-based controller.

We will also need the following result to characterize the properties of the Lyapunov-based controller. By continuity and the local Lipschitz property assumed for the vector field $f(x, u, w)$ and the continuous differentiable property of the control Lyapunov function $V$, there exist positive constants $M$, $L_{w}$ and $L_{x}$ such that

$$
\begin{array}{r}
|f(x, h(x), w)| \leq M \\
\left|\frac{\partial V}{\partial x} f(x, h(x), w)-\frac{\partial V}{\partial x} f\left(x^{\prime}, h(x), 0\right)\right| \leq L_{w}|w|+L_{x}\left|x-x^{\prime}\right|
\end{array}
$$

for all $x, x^{\prime} \in \Omega_{\rho}$ and $w \in W$. These constants will be used to bound the evolution of the Lyapunov function for the closedloop system, when the controller $h(x)$ is applied in a sampleand-hold scheme in the presence of data losses.

Existing LMPC schemes [33]-[35] that do not take into account data losses are defined by the following optimization problem

$$
\begin{aligned}
\min _{u \in S(\Delta)} & \int_{t_{k}}^{t_{k+N}}\left[\tilde{x}(\tau)^{T} Q_{c} \tilde{x}(\tau)+u(\tau)^{T} R_{c} u(\tau)\right] d \tau \\
\text { s.t. } & \dot{\tilde{x}}(t)=f(\tilde{x}(t), u(t), 0) \\
& \tilde{x}\left(t_{k}\right)=x\left(t_{k}\right) \\
& \left.\dot{V}\left(x\left(t_{k}\right)\right)\right|_{u\left(t_{k}\right)} \leq\left.\dot{V}\left(x\left(t_{k}\right)\right)\right|_{h\left(x\left(t_{k}\right)\right)}
\end{aligned}
$$

where $S(\Delta)$ is the family of piece-wise constant functions with sampling period $\Delta, \tilde{x}(t)$ is the predicted trajectory of the nominal system for the input trajectory computed by the LMPC, and $Q_{c}, R_{c}$ are positive definite weight matrices that define the cost. At each sampling time $t_{k}$, the new state $x\left(t_{k}\right)$ is received from the sensors, problem (5) is solved, and $u\left(t_{k}\right)$ is applied to the closed-loop system for $t \in\left[t_{k} t_{k+1}\right]$. This is the standard receding horizon strategy and it does not take into account that the state might not be available at a given sampling time due to data losses. Constraint (5d) guarantees that the value of the time derivative of the Lyapunov function at $t_{k}$ based on the nominal model is smaller or equal to the value obtained if the Lyapunov-based controller $u=h(x)$ is implemented in the closed-loop system. This is the contractive constraint that allows one to prove (when no data losses are taken into account) that the LMPC inherits the stability and robustness properties of the Lyapunov-based controller $h(x)$. In Section IV we present the main contribution of this paper, a novel LMPC controller based on a contractive constraint and a receding horizon implementation scheme that take explicitly into account data losses. The main property of the LMPC controller (5) is that the origin of the closed loop system is practically stable for all initial states inside the stability region $\Omega_{\rho}$ for sufficiently small sampling time $\Delta$ and sufficiently small disturbance upper bound $\theta$. This property is also guaranteed by the Lyapunov-based controller when 
this controller is applied in a sample-and-hold fashion, see [48], [49] for results on sampled-data systems. The main advantage of LMPC approaches with respect to the Lyapunov-based controller, is that optimality considerations can be taken explicitly into account (as well as constraints on the inputs and the states [34]) in the computation of the controller within an on-line optimization framework, improving the closed-loop performance of the system.

\section{Data Losses and Asynchronous Sampling}

In the following sections, we will refer to data losses as the source of asynchronous behaviour of the processes under consideration. Note that asynchronous sampling with respect to a fixed sampling can be viewed as data losses with respect to the regular measurement schedule. All the results presented can be applied to systems subject to asynchronous measurement sampling.

To model data losses, an auxiliary random variable $s\left(t_{k}\right)$ is introduced with $t_{k}=t_{0}+k \Delta$ where $t_{0}$ is the initial time and $k=0,1 \ldots$ Data may be lost in both the sensor-controller link and the controller-actuator link. When $s\left(t_{k}\right)=1$, at sampling time $t_{k}$ the full state vector is available for the controller and the actuator receives the new data. When $s\left(t_{k}\right)=0$, either the measurement of the full state is not available, or the new input trajectory evaluated is not received by the actuator. In both cases, the actuator has to operate in open-loop so, from a control point of view, both cases are equivalent. The probability distribution of variable $s\left(t_{k}\right)$ characterizes the quality of the communication links. In general, if the probability of successful communication is fixed or if the data losses are modeled using a random variable governed by a Markov chain, there exists the possibility of arbitrarily large (but finite) periods of time without feedback. In this case, it is not possible to provide guaranteed stability properties, because there exists a non-zero probability that the system operates in open-loop for a period of time large enough for the state to leave the stability region or even diverge to infinity during that period of time (i.e., finite escape time). In order to study the stability properties in a deterministic framework, in this paper we consider systems where there is a limit on the maximum number of consecutive sampling times in which data is lost, i.e.,

$$
\begin{aligned}
& N_{o} \geq \max j-i \\
& \quad \text { s.t. } s\left(t_{i}\right)=s\left(t_{j}\right)=1, \quad s\left(t_{k}\right)=0, \quad k \in(i, j) .
\end{aligned}
$$

This bound on the maximum period of time in which the loop is open has been also used in other works in the literature [20]-[22], [12] and allows us to study deterministic notions of stability.

When data losses occur, most approaches set the control input to zero or to the last implemented value. Instead, when data is lost, we take advantage of the model predictive control scheme to update the input based on a prediction obtained using the model. This is achieved using the following scheme: At each sampling time where no data is lost (i.e., the current state is available and the controller can send information to the actuator), the Lyapunov-based controller uses the nominal model of the system (system (1) with $w(t) \equiv 0$ for all $t$ ) to predict the future trajectory $\tilde{x}(t)$ for a given input trajectory $u(t) \in$
$S(\Delta)$ with $t \in\left[t_{k}, t_{k+N}\right]$ where $N$ is the prediction horizon. A cost function is minimized, while assuring that the value of the Lyapunov function along the predicted trajectory $\tilde{x}(t)$ satisfies a Lyapunov-based contractive constraint. The whole optimal input trajectory $u(t)$, with $t \in\left[t_{k}, t_{k+N}\right]$, is sent to the actuator which implements the first value, and stores the rest (note that this implies higher bandwidth requirements). When the state is not available, or the data sent from the controller to the actuator is lost, the actuator keeps implementing the last received optimal trajectory. If data is lost for a period larger than the prediction horizon, the actuator sets the input to the last implemented value or to a fixed value. The proposed implementation technique is described in Section IV.

Remark 1: The data losses model proposed is based on full state transmission. This is different from the formulation of Walsh et al. [20], [21]. For this reason, we do not consider any transmission protocol of the kind introduced by [22].

Remark 2: The definition of $N_{o}$ in (6) is reminiscent of the maximum allowable transmission interval (MATI) introduced by Walsh et al. [20], [21]. Specifically, MATI refers to the maximum time between any two consecutive transmissions (which may include part of the full state vector), while $N_{o} \Delta$ refers to the maximum time between any two successful transmissions of the full state vector to the controller and the entire input trajectory from the controller to the actuator.

\section{PROPERTIES OF THE LyAPUNOV-BASED CONTROLLER}

In this section, we present results that will be used to prove the stability and robustness properties of the LMPC controller proposed in Section IV. Specifically, we establish certain stability and robustness properties of the Lyapunov-based controller $u=$ $h(x)$ in the presence of uncertainty and data losses.

\section{A. Nominal Closed-Loop System Under Sample-and-Hold Control}

We first investigate the properties of the Lyapunov-based controller when applied in a sample-and-hold fashion without taking into account uncertainty or data losses. These properties are important because the proposed LMPC scheme is based on the nominal model, that is, system (1) with $w(t) \equiv 0$. Note that the sampled trajectories may fail to be defined on all times because of the possibility of finite escape time in one of the intervals. In the results presented in this work however, the sampling time is chosen such that the trajectories are well defined (i.e., $x(t)$ is continuous and bounded for all times). To state our results we need the following definition:

Definition 1: The nominal sampled trajectory of system (1) associated with a feedback law $h(x)$ with sampling time $\Delta$ starting at $x\left(t_{0}\right)$ is denoted by $\hat{x}(t)$ and is obtained by solving recursively

$$
\dot{\hat{x}}(t)=f\left(\hat{x}(t), h\left(\hat{x}\left(t_{k}\right)\right), 0\right), \quad t \in\left[t_{k}, t_{k+1}\right]
$$

where $t_{k}=t_{0}+k \Delta, k=0,1, \ldots$ and $\hat{x}\left(t_{0}\right)=x\left(t_{0}\right)$.

In the sampled trajectory of the nominal closed-loop system, the uncertainty is set equal to zero. The LMPC scheme to be proposed in Section IV below optimizes a cost function, subject to a set of constraints defined by the nominal sampled trajectory of system (1). This allows us to formulate an LMPC problem 
that does not depend on the uncertainty and so it is of manageable computational complexity.

The Lyapunov-based controller possesses a robustness property in the sense that it maintains practical stability of system (1) under a sample-and-hold implementation if some conditions are satisfied. The following proposition states that there exists a sufficiently small sampling time $\Delta$ and a positive constant $\rho_{s}$ such that if at time $t_{k}$ the state is inside $\Omega_{\rho}$ but outside $\Omega_{\rho_{s}}$, then the Lyapunov function will decrease, that is, $V\left(\hat{x}\left(t_{k+1}\right)\right)<$ $V\left(\hat{x}\left(t_{k}\right)\right)$. Using this result it can be proved that the system converges to $\Omega_{\rho_{s}}$ in finite time from any initial state inside $\Omega_{\rho}$.

Proposition 1: Consider the nominal sampled trajectory $\hat{x}(t)$ of system (1) for a controller $h(x)$ that satisfies (2). Let $\Delta, \epsilon_{s}>$ 0 and $\rho>\rho_{s}>0$ satisfy

$$
-\alpha_{3}\left(\alpha_{2}^{-1}\left(\rho_{s}\right)\right)+\alpha_{4}\left(\alpha_{1}^{-1}(\rho)\right) L_{x} M \Delta \leq-\epsilon_{s} / \Delta .
$$

Then, for any $k$, if $\hat{x}\left(t_{k}\right) \in \Omega_{\rho} / \Omega_{\rho_{s}}$, the following inequalities hold:

$$
\begin{aligned}
& V\left(\hat{x}\left(t_{k+1}\right)\right) \leq V\left(\hat{x}\left(t_{k}\right)\right)-\epsilon_{s} \\
& V(\hat{x}(t)) \leq V\left(\hat{x}\left(t_{k}\right)\right), \quad \forall t \in\left[t_{k}, t_{k+1}\right] .
\end{aligned}
$$

Proof: Following Definition 1, the time derivative of the Lyapunov function along the nominal sampled trajectory $\hat{x}(t)$ of system (1) in $t \in\left[t_{k}, t_{k+1}\right]$ is given by

$$
\dot{V}(\hat{x}(t))=\frac{\partial V}{\partial x} f\left(\hat{x}(t), h\left(\hat{x}\left(t_{k}\right)\right), 0\right) .
$$

Adding and subtracting $\frac{\partial V}{\partial x} f\left(\hat{x}\left(t_{k}\right), h\left(\hat{x}\left(t_{k}\right)\right), 0\right)$ and taking into account (2) we obtain

$$
\begin{aligned}
\dot{V}(\hat{x}(t)) \leq & -\alpha_{3}\left(\left|\hat{x}\left(t_{k}\right)\right|\right)+\frac{\partial V}{\partial x} f\left(\hat{x}(t), h\left(\hat{x}\left(t_{k}\right)\right), 0\right) \\
& -\frac{\partial V}{\partial x} f\left(\hat{x}\left(t_{k}\right), h\left(\hat{x}\left(t_{k}\right)\right), 0\right) .
\end{aligned}
$$

From (2) we have that

$$
\begin{gathered}
\left|\frac{\partial V}{\partial x}\right| \leq \alpha_{4}\left(\alpha_{1}^{-1}(\rho)\right) \\
-\alpha_{3}\left(\left|\hat{x}\left(t_{k}\right)\right|\right) \leq-\alpha_{3}\left(\alpha_{2}^{-1}\left(\rho_{s}\right)\right)
\end{gathered}
$$

for all $\hat{x}\left(t_{k}\right) \in \Omega_{\rho} / \Omega_{\rho_{s}}$. Substituting (4) and (11) into (10), the following bound for $\dot{V}(\hat{x}(t))$ is obtained

$$
\dot{V}(\hat{x}(t)) \leq-\alpha_{3}\left(\alpha_{2}^{-1}\left(\rho_{s}\right)\right)+\alpha_{4}\left(\alpha_{1}^{-1}(\rho)\right) L_{x}\left|\hat{x}(t)-\hat{x}\left(t_{k}\right)\right| .
$$

Taking into account (3) and the continuity of $x(t)$ and $\hat{x}(t)$, the following bound can be written for all $t \in\left[t_{k}, t_{k+1}\right]$

$$
\left|\hat{x}(t)-\hat{x}\left(t_{k}\right)\right| \leq M \Delta \text {. }
$$

Using this expression, we obtain the following bound on the time derivative of the Lyapunov function for $t \in\left[t_{k}, t_{k+1}\right]$, for all initial states $\hat{x}\left(t_{k}\right) \in \Omega_{\rho} / \Omega_{\rho_{s}}$

$$
\dot{V}(\hat{x}(t)) \leq-\alpha_{3}\left(\alpha_{2}^{-1}\left(\rho_{s}\right)\right)+\alpha_{4}\left(\alpha_{1}^{-1}(\rho)\right) L_{x} M \Delta .
$$

If (7) is satisfied, then $\dot{V}(\hat{x}(t)) \leq-\epsilon_{s} / \Delta$. Integrating this bound on $t \in\left[t_{k}, t_{k+1}\right]$ we obtain that the inequalities of (8) hold.

Proposition 1 guarantees that if system (1) under the control law $u=h(x)$, implemented in a sample-and-hold fashion, starts in $\Omega_{\rho} / \Omega_{\rho_{s}}$, it reaches $\Omega_{\rho_{s}}$ provided that $\Delta$ is sufficiently small. However, $\Omega_{\rho_{s}}$ is not proved to be an invariant set for $\hat{x}(t)$. For continuous-time systems under continuous control implementation, a sufficient condition for invariance is that the derivative of the Lyapunov function is negative on the boundary of the set. For systems with continuous-time dynamics and sample-and-hold control implementation this condition is not sufficient because the time derivative may become positive during the sampling period and the system may leave the set before a new sample is obtained. The following proposition defines a region that is invariant. The region is defined by $\rho_{\min }$, which is the maximum value that the Lyapunov function can achieve in a time period of length $\Delta$ when $\hat{x}(0) \in \Omega_{\rho_{s}}$. The result is given in terms of a function that upper bounds the Lyapunov function along the nominal sampled trajectory.

Proposition 2: Consider the nominal sampled trajectory $\hat{x}(t)$ of system (1) for a controller $h(x)$ that satisfies (2). Let $\Delta, \epsilon_{s}>$ 0 and $\rho>\rho_{s}>0$ satisfy (7). Then, if $\rho_{\min } \leq \rho$ where

$$
\rho_{\min }=\max \left\{V(\hat{x}(t+\Delta)): V(\hat{x}(t)) \leq \rho_{s}\right\}
$$

and $\hat{x}\left(t_{0}\right) \in \Omega_{\rho}$, the following inequalities hold

$$
\begin{aligned}
& V\left(\hat{x}\left(t_{k}\right)\right) \leq \max \left\{V\left(\hat{x}\left(t_{0}\right)\right)-k \epsilon_{s}, \rho_{\min }\right\} \\
& V(\hat{x}(t)) \leq \max \left\{V\left(\hat{x}\left(t_{k}\right)\right), \rho_{\min }\right\}, \quad \forall t \in\left[t_{k}, t_{k+1}\right] .
\end{aligned}
$$

Proof: Applying Proposition 1 recursively, if $\hat{x}\left(t_{0}\right) \in$ $\Omega_{\rho} / \Omega_{\rho_{s}}$ there exists $k^{*}>0$ such that $\hat{x}\left(t_{k^{*}}\right) \in \Omega_{\rho_{s}}$, $\hat{x}\left(t_{k}\right) \in \Omega_{\rho} / \Omega_{\rho_{s}}, \forall k \leq k^{*}$ and $V\left(\hat{x}\left(t_{k}\right)\right) \leq V\left(\hat{x}\left(t_{0}\right)\right)-k \epsilon_{s}$. Once the state converges to $\Omega_{\rho_{s}} \subset \Omega_{\rho_{\min }}$ (or starts there) it remains inside $\Omega_{\rho_{\min }}$ for all times. This statement holds because by definition of $\rho_{\min }$, if $\hat{x}\left(t_{k}\right) \in \Omega_{\rho_{s}}$ then $\hat{x}\left(t_{k+1}\right) \in \Omega_{\rho_{\min }}$. It follows that (12) holds and that $\hat{x}(t)$ is ultimately bounded in $\Omega_{\rho_{\min }}$. The bound on the evolution of the state between sampling times follow from Proposition 1.

\section{B. Effect of the Uncertainty}

In this section we study the closed-loop trajectories of system (1) with controller $u=h(x)$ implemented in a sample-andhold fashion taking into account uncertainty. We assume that there are no data losses. The results presented in this section are obtained using standard techniques used to study sampled-data systems [48], [49], nonlinear systems subject to data losses [1], [21], [50], [22] and more recently scheduling of control tasks [51]. The main idea is to characterize the conditions under which the Lyapunov function is guaranteed to be decreasing between sampling times for any given uncertainty realization. We next define the open-loop sampled trajectory of system (1).

Definition 2: The open-loop sampled trajectory of system (1) associated with a feedback law $h(x)$ with sampling time $\Delta$ starting at $x\left(t_{0}\right)$ for a given disturbance trajectory $w(t)$ is denoted $x(t)$ and is obtained by solving

$$
\dot{x}(t)=f\left(x(t), h\left(\hat{x}\left(t_{k}\right)\right), w(t)\right), \quad t \in\left[t_{k}, t_{k+1}\right]
$$


where $t_{k}=t_{0}+k \Delta, k=0,1, \ldots$ and $\hat{x}(t)$ is the nominal sampled trajectory of system (1) associated with a feedback law $h(x)$ with sampling time $\Delta$ starting at $x\left(t_{0}\right)$.

In this section, we will use the open-loop sampled trajectory to characterize the properties of the Lyapunov-based controller when no data is lost. This is done studying the evolution of the open-loop sampled trajectory in the first sampling step, i.e., $x(t)$ with $t \in\left[t_{0}, t_{1}\right]$. In this time period the control input is evaluated utilizing the actual state (because $\hat{x}\left(t_{0}\right)=x\left(t_{0}\right)$ ). In this way, we can apply recursively the obtained properties to characterize the closed-loop behavior of the system in the presence of uncertainty. The properties of the Lyapunov-based controller in the presence of data losses will be studied in the next subsection.

Proposition 3: Consider the open-loop sampled trajectory $x(t)$ of system (1) for a controller $h(x)$ that satisfies (2). Let $\Delta, \epsilon_{s}, \epsilon_{w}, \theta>0$ and $\rho>\rho_{s}>0$ satisfy (7) and

$$
-\epsilon_{s}+\alpha_{4}\left(\alpha_{1}^{-1}(\rho)\right) \gamma_{e}(\Delta)+\beta \gamma_{e}(\Delta)^{2} \leq-\epsilon_{w}
$$

with $\gamma_{e}(\Delta)=\left(L_{w} \theta / L_{x}\right)\left(e^{L_{x} \Delta}-1\right)$. Then, if $x\left(t_{0}\right) \in \Omega_{\rho} / \Omega_{\rho_{s}}$, the following inequalities hold for all $w(t) \in W$

$$
\begin{aligned}
V\left(x\left(t_{1}\right)\right) & \leq V\left(x\left(t_{0}\right)\right)-\epsilon_{w}, \\
V(x(t)) & \leq V\left(x\left(t_{0}\right)\right), \quad \forall t \in\left[t_{0}, t_{1}\right] .
\end{aligned}
$$

Proof: Since the Lyapunov function $V(x)$ is continuous and bounded on compact sets, there exists a positive constant $\beta$ such that a Taylor series expansion of $V$ around $\hat{x}$ yields

$$
V(x) \leq V(\hat{x})+\frac{\partial V}{\partial x}|x-\hat{x}|+\beta|x-\hat{x}|^{2}, \quad \forall x, \hat{x} \in \Omega_{\rho} .
$$

Note that the term $\beta|x-\hat{x}|^{2}$ bounds the high order terms of the Taylor series of $V(x)$ for $x, \hat{x} \in \Omega_{\rho}$. Taking into account (2) the following bound for $V(x)$ is obtained

$V(x) \leq V(\hat{x})+\alpha_{4}\left(\alpha_{1}^{-1}(\rho)\right)|x-\hat{x}|+\beta|x-\hat{x}|^{2}, \forall x, \hat{x} \in \Omega_{\rho}$.

We define the error vector as $e(t)=x(t)-\hat{x}(t)$. The derivative of the error is given by

$$
\dot{e}(t)=f(x(t), h(\hat{x}(t)), w(t))-f(\hat{x}(t), h(\hat{x}(t)), 0)
$$

so by (4)

$$
|\dot{e}(t)| \leq L_{w}|w(t)|+L_{x}|x(t)-\hat{x}(t)| \leq L_{w} \theta+L_{x}|e(t)|
$$

for all $x(t), \hat{x}(t) \in \Omega_{\rho}$ all $w(t) \in W$. Integrating $|\dot{e}(t)|$ with $e\left(t_{0}\right)=0$ (recall that $\hat{x}\left(t_{0}\right)=x\left(t_{0}\right)$ ), the following bound on the norm of the error vector is obtained

$$
|e(t)| \leq \frac{L_{w} \theta}{L_{x}}\left(e^{L_{x}\left(t-t_{0}\right)}-1\right) .
$$

Using this bound we can define a function of class $\mathcal{K}, \gamma_{e}(\cdot)$, such that

$$
|x(t)-\hat{x}(t)| \leq \gamma_{e}\left(t-t_{0}\right):=\frac{L_{w} \theta}{L_{x}}\left(e^{L_{x}\left(t-t_{0}\right)}-1\right)
$$

for all $t>t_{0}$. Using (15) and (16), the following bound on $V\left(x\left(t_{k}\right)\right)$ is obtained

$V\left(x\left(t_{k}\right)\right) \leq V\left(\hat{x}\left(t_{k}\right)\right)+\alpha_{4}\left(\alpha_{1}^{-1}(\rho)\right) \gamma_{e}(k \Delta)+\beta \gamma_{e}(k \Delta)^{2} .(17)$
Using Proposition 1 the following inequality holds for all $\hat{x}\left(t_{0}\right) \in \Omega_{\rho} / \Omega_{\rho_{s}}$

$$
V\left(\hat{x}\left(t_{1}\right)\right) \leq V\left(\hat{x}\left(t_{0}\right)\right)-\epsilon_{s} .
$$

Using (18) and taking into account that $x\left(t_{0}\right)=\hat{x}\left(t_{0}\right)$, (17) yields

$$
V\left(x\left(t_{1}\right)\right) \leq V\left(x\left(t_{0}\right)\right)-\epsilon_{s}+\alpha_{4}\left(\alpha_{1}^{-1}(\rho)\right) \gamma_{e}(\Delta)+\beta \gamma_{e}(\Delta)^{2}
$$

for all $w(t) \in W$. If (13) is satisfied then both inequalities of (14) hold because the increase of the bound on the Lyapunov function due to the error between the actual trajectory and the nominal trajectory is a strictly increasing function of time.

The above proposition characterizes the trajectory of the first time step of the open-loop sampled trajectory for initial states $x\left(t_{0}\right)$ inside the ring $\Omega_{\rho} / \Omega_{\rho_{s}}$. In the following proposition we study the trajectory of the first time step of the open-loop sampled trajectory for any initial state $x\left(t_{0}\right)$ inside the set $\Omega_{\rho}$. This proposition takes into account that the value of the Lyapunov function of the closed-loop sampled trajectories that start inside the inner set $\Omega_{\rho_{s}}$ may increase.

Proposition 4: Consider the open-loop sampled trajectory $x(t)$ of system (1) under the controller $h(x)$ that satisfies (2). Let $\Delta, \rho, \rho_{s}, \epsilon_{s}, \epsilon_{w}$ and $\theta$ satisfy (7) and (13). Then, if $\rho^{*} \leq \rho$ where

$$
\rho^{*}=\rho_{\min }+\alpha_{4}\left(\alpha_{1}^{-1}(\rho)\right) \gamma_{e}(\Delta)+\beta \gamma_{e}(\Delta)^{2}
$$

and $x\left(t_{0}\right) \in \Omega_{\rho}$, the following inequalities hold for all $w(t) \in$ $W$

$$
\begin{aligned}
& V\left(x\left(t_{1}\right)\right) \leq \max \left\{V\left(x\left(t_{0}\right)\right)-\epsilon_{w}, \rho^{*}\right\} \\
& V(x(t)) \leq \max \left\{V\left(x\left(t_{0}\right)\right), \rho^{*}\right\}, \forall t \in\left[t_{0}, t_{1}\right] .
\end{aligned}
$$

Proof: Since $\hat{x}\left(t_{0}\right)=x\left(t_{0}\right)$, Proposition 2 guarantees that

$$
\begin{gathered}
V\left(\hat{x}\left(t_{1}\right)\right) \leq \max \left\{V\left(\hat{x}\left(t_{0}\right)\right)-\epsilon_{s}, \rho_{\min }\right\} \\
V(\hat{x}(t)) \leq \max \left\{V\left(\hat{x}\left(t_{0}\right)\right), \rho_{\min }\right\}, \quad \forall t \in\left[t_{k}, t_{k+1}\right] .
\end{gathered}
$$

Using (17) we obtain that

$V(x(t)) \leq V(\hat{x}(t))+\alpha_{4}\left(\alpha_{1}^{-1}(\rho)\right) \gamma_{e}\left(t-t_{0}\right)+\beta \gamma_{e}\left(t-t_{0}\right)^{2}$.

Applying (19) and (13) for $t-t_{0}=\Delta$, we obtain that the inequalities of (20) hold.

When no data losses are present, at each time step, the input is computed utilizing the actual state, so Proposition 4 can be used recursively. These results will be used in Section IV to prove the main contributions of this work.

\section{Data Losses}

In this section, we study the stability properties of system (1) in closed-loop with controller $h(x)$ applied in a sampleand-hold fashion in the presence of data losses. The trajectory of system (1) in the presence of data losses (i.e., the actual state is not available for the Lyapunov-based controller to compute its control action) when the nominal model is used to estimate the state of the system and update the control input, is defined by the open-loop sampled trajectory (see Definition 2). 
Specifically, when data is lost, the state of the system is estimated using the nominal model and the input is decided using this estimated state. In this case, the control system is operating in open-loop because the input, is not decided on behalf of the actual state $x(t)$, but instead is computed by using the estimated state $\hat{x}(t)$ provided by the sampled-nominal trajectory. When data is lost, the initial state $x\left(t_{0}\right)$ of the open-loop sampled trajectory is given by the last received state. We evaluate the nominal sampled trajectory $\hat{x}(t)$ from this initial state $x\left(t_{0}\right)$. This trajectory is used by the actuator which implements, $u(t)=h\left(\hat{x}\left(t_{k}\right)\right), t \in\left[t_{k}, t_{k+1}\right]$ until a new measurement of the actual state is received. We will study this trajectory to characterize the stability properties of the Lyapunov-based controller in the presence of uncertainty and data losses. The LMPC will inherit these properties because this strategy is consistent with the modified receding horizon scheme proposed in the previous section.

Due to the effect of the uncertainties, there is an error between the actual state $x(t)$ and the estimated state $\hat{x}(t)$. This error limits the maximum amount of uncertainty the system can handle and the maximum time the system can operate in open-loop without leaving the stability region $\Omega_{\rho}$. The result presented in Proposition 5 below states that under certain conditions if the system starts inside $\Omega_{\rho}$, the open-loop sampled trajectory will remain inside this set for a certain period of time and provides an upper bound on the estimate of this time in terms of the system and controller parameters. This result is used in the following section to characterize the stability of the closed-loop system under LMPC in the presence of data losses.

Proposition 5: Consider the open-loop sampled trajectory $x(t)$ of system (1) for a controller $h(x)$ that satisfies (2). Let $\Delta$, $\rho, \rho_{s}, \epsilon_{s}, \epsilon_{w}$ and $\theta$ satisfy (7), (13) and (19). Then there exists integer $N_{R} \geq 1$ such that if $x\left(t_{0}\right) \in \Omega_{\rho}$ and is known (see Definition 2), then $x(t) \in \Omega_{\rho} \forall t \in\left[t_{0}, t_{N_{R}}\right]$ and $\forall w(t) \in W$.

Proof: The proof is based on using (17) to obtain a bound on the worst case evolution of $x\left(t_{k}\right)$ based on the trajectory of the nominal system $\hat{x}\left(t_{k}\right)$. Following Proposition 2 we know that

$$
V\left(\hat{x}\left(t_{k}\right)\right) \leq \max \left\{V\left(\hat{x}\left(t_{0}\right)\right)-k \epsilon_{s}, \rho_{\min }\right\}
$$

and that, because the upper bound used to obtain this inequality is a strictly increasing function of time, $V(\hat{x}(t)) \leq$ $V\left(\hat{x}\left(t_{k}\right)\right), \forall t \leq t_{k}$. To evaluate the maximum $N_{R}$ such that $x\left(t_{N_{R}}\right) \in \Omega_{\rho}$ we must take into account both possible values of the bound on $V\left(\hat{x}\left(t_{k}\right)\right)$. The proof consists of two parts. We first evaluate $N_{1} \geq 1$ such that if $V\left(\hat{x}\left(t_{k}\right)\right) \leq V\left(\hat{x}\left(t_{0}\right)\right)-k \epsilon_{s}$ for all $k$ then $x\left(t_{N_{1}}\right) \in \Omega_{\rho}$. Then we define $N_{2} \geq 1$ such that if $V\left(\hat{x}\left(t_{k}\right)\right) \leq \rho_{\min }$ for all $k$ then $x\left(t_{N_{2}}\right) \in \Omega_{\rho}$. It follows that $N_{R}=\min \left\{N_{1}, N_{2}\right\} \geq 1$ satisfies the proposition.

Part 1: Assume that $V\left(\hat{x}\left(t_{k}\right)\right) \leq V\left(\hat{x}\left(t_{0}\right)\right)-k \epsilon_{s}$ for all $k$. A sufficient condition to prove that if $x\left(t_{0}\right) \in \Omega_{\rho}$ then $x(t) \in$ $\Omega_{\rho} \forall t \in\left[t_{0}, t_{N_{1}}\right]$ is that if $V\left(x\left(t_{0}\right)\right)=\rho$ then $x(t) \in \Omega_{\rho} \forall t \in$ $\left[t_{0}, t_{N_{1}}\right]$. Using (17), the following bound on $V(x(t))$ is obtained for states on the boundary of $\Omega_{\rho}$

$$
V\left(x\left(t_{k}\right)\right) \leq \rho-k \epsilon_{s}+\alpha_{4}\left(\alpha_{1}^{-1}(\rho)\right) \gamma_{e}(k \Delta)+\beta \gamma_{e}(k \Delta)^{2} .
$$

This bound is defined as the sum of a strictly decreasing function and a strictly increasing function of time. The con- stant $N_{1}$ is defined as the maximum $k$ such that $V\left(x\left(t_{k}\right)\right) \leq \rho$, i.e.,

$$
N_{1} \epsilon_{s} \geq \alpha_{4}\left(\alpha_{1}^{-1}(\rho)\right) \gamma_{e}\left(N_{1} \Delta\right)+\beta \gamma_{e}\left(N_{1} \Delta\right)^{2} .
$$

If $V\left(x\left(t_{N_{1}}\right)\right) \leq \rho$, then it holds that $x(t) \in \Omega_{\rho}$ for all $t \in\left[t_{0}, t_{N_{1}}\right]$. A necessary and sufficient condition for the existence of a constant $N_{1} \geq 1$ that satisfies this inequality is that $V\left(x\left(t_{0}\right)\right) \geq V\left(x\left(t_{1}\right)\right)$. This holds because (13) is satisfied.

Part 2: Assume that $\hat{x}\left(t_{k}\right) \in \Omega_{\rho_{\min }}$ for all $k$. Following the same reasoning as in part 1 of the proof of this proposition, and using (17), the following bound is obtained

$$
V\left(x\left(t_{k}\right)\right) \leq \rho_{\min }+\alpha_{4}\left(\alpha_{1}^{-1}(\rho)\right) \gamma_{e}(k \Delta)+\beta \gamma_{e}(k \Delta)^{2} .
$$

This bound is strictly increasing with respect to $k$. Constant $N_{2}$ is defined as the maximum $k$ such that $V\left(x\left(t_{k}\right)\right) \leq \rho$, i.e.,

$$
\rho-\rho_{\min } \geq \alpha_{4}\left(\alpha_{1}^{-1}(\rho)\right) \gamma_{e}\left(N_{2} \Delta\right)+\beta \gamma_{e}\left(N_{2} \Delta\right)^{2} .
$$

If $V\left(x\left(t_{N_{2}}\right)\right) \leq \rho$, then it holds that $x(t) \in \Omega_{\rho}$ for all $t \in$ $\left[t_{0}, t_{N_{2}}\right]$. Because (19) is satisfied, it follows that $V\left(x\left(t_{1}\right)\right) \leq \rho$. This assures the existence of $N_{2} \geq 1$ that satisfies (21). Note that (17) is valid only if $x(t), \hat{x}(t) \in \Omega_{\rho}$ for all $t \in\left[t_{0}, t_{N_{R}}\right]$. The first claim $\left(x(t) \in \Omega_{\rho}\right)$ holds by the definition of $N_{R}$ (note that this is exactly what the proposition states). The second claim $\left(\hat{x}(t) \in \Omega_{\rho}\right)$ holds because of Proposition 2 .

The results presented in this section characterize the stability and robustness properties of the Lyapunov-based controller. Proposition 4 guarantees practical stability in the absence of data losses, and Proposition 5 guarantees that the stability region is invariant if the maximum time without measurements is shorter than a fixed value. These properties will be inherited by the proposed LMPC scheme, which at the same time, introduces optimality considerations improving closed-loop performance. This result is presented in the following section.

Remark 3: Proposition 5 guarantees that the stability region is invariant if the maximum time without measurements is shorter than $N_{R} \Delta$ and provides an estimate of the constant $N_{R}$ that is guaranteed to be greater or equal than 1 . Note that if $N_{R}$ is equal to 1 in order to guarantee closed-loop stability, a measurement must be available every sampling time, so in practice, no data losses can occur. This is possible, because $N_{R}$ depends on the parameters of the system, the Lyapunov-based controller, the sampling time $\Delta$ and the size of the uncertainty $\theta$. For a particular choice of $\Delta$ and $\theta$, the closed-loop system without data losses may be guaranteed to be practically stable, so $N_{R}=1$ guarantees that the stability region is invariant; but in the presence of data losses, it might not be possible to guarantee the same property for $N_{R}>1$.

Remark 4: Although the proofs of propositions 1, 2, 3, 4 and 5 are constructive, the constants obtained are conservative. This is the case with most of the results of the type presented in this paper, see for example [49], [51] for further discussion on this issue. In practice, the sampling time and the maximum time that the system can operate in open-loop without leaving the closedloop stability region are better estimated through closed-loop simulation. The various inequalities proved in these five propositions are more useful as guidelines on the interaction between the various parameters that define the system and the controller 
and may be used as guidelines to design the controller and the network.

\section{Proposed Lyapunov-Based Model PREDICTIVE CONTROL}

In this section, a new Lyapunov-based model predictive controller for system (1) is proposed which takes into account explicitly data losses, both in the constraints imposed in the optimization problem and in the implementation procedure. Previous LMPC schemes (see [33]-[35]) are based on problem (5). When data losses do not occur, these controllers guarantee practical stability (due to the sample-and-hold implementation). When data losses are taken into account whether the actuator keeps the input at the last value, sets the input to a constant value, or keeps on implementing the previously evaluated input trajectory (like we propose for the new LMPC controller), the existing LMPC schemes cannot guarantee closed-loop stability. In particular, there are no guarantees that the LMPC optimization problem will be feasible for all times, i.e., that the state will remain inside the stability region for all time. The contractive constraint (5d) only takes into account the first time step and does not give any information on the behavior of the system if data losses occur. If no additional constraints are included in the optimization problem, no claims on the closed-loop stability behavior of the system can be made. For this reason, when data losses are taken into account, the constraints of the LMPC problem have to be modified. The proposed LMPC that takes into account data losses in an explicit way is based on the following finite horizon constrained optimal control problem

$$
\begin{aligned}
\min _{u \in S(\Delta)} & \int_{t_{k}}^{t_{k+N}}\left[\tilde{x}(\tau)^{T} Q_{c} \tilde{x}(\tau)+u(\tau)^{T} R_{c} u(\tau)\right] d \tau \\
\text { s.t. } & \dot{\tilde{x}}(t)=f(\tilde{x}(t), u(t), 0) \\
& \tilde{x}\left(t_{k}\right)=x\left(t_{k}\right) \\
& V(\tilde{x}(t)) \leq V(\hat{x}(t)), \quad \forall t \in\left[t_{k}, t_{k+N_{R}}\right]
\end{aligned}
$$

where $S(\Delta)$ is the family of piece-wise constant functions with sampling period $\Delta, \tilde{x}(t)$ is the predicted sampled trajectory of the nominal system for the input trajectory computed by the LMPC (22), $\hat{x}(t)$ is the nominal sampled trajectory under the Lyapunov-based controller $u=h(\hat{x}(t))$ (see Definition 1), and $Q_{c}, R_{c}$ are weight matrices that define the cost. This optimization problem does not depend on the uncertainty and assures that the LMPC inherits the properties of the Lyapunov-based controller. To take full advantage of the use of the nominal model in the computation of the control action, we take $N \geq N_{R}$.

The main difference between constraint (5d) and constraint (22d) is that under the assumption of flawless communications, the optimization problem is solved at each sampling time. This implies, that to prove that the LMPC inherits the stability properties of the Lyapunov-based controller it is sufficient to guarantee that the LMPC controller provides at least the same decrease of the Lyapunov function as the Lyapunov based controller in the first time step. When data losses are taken into account, in order to prove that the LMPC inherits the same properties of the Lyapunov-based controller applied in a sample and hold scheme and using the model to predict the evolution of the system when no data losses are present, the constraint must hold along the whole prediction horizon. In this manner, when data is lost, the optimal input trajectory evaluated guarantees that the predicted decrease of the Lyapunov function using the nominal model is at least equal to the one obtained applying the Lyapunov-based controller, and hence, the same robustness properties are inherited.

The implementation procedure is modified to profit from the last optimal input trajectory when data is lost. At each sampling time, if the current state $x\left(t_{k}\right)$ is available and belongs in $\Omega_{\rho}$, the controller solves the optimization problem, obtaining a new optimal input trajectory $u_{k}^{*}(t), t \in\left[t_{k}, t_{k+N}\right]$. This input trajectory is sent to the actuator through the communication link. If the actuator receives the new trajectory (that is, if the loop is closed), it implements the first input and stores the rest in memory. If either the sensor-controller link or the controller-actuator link fail, the actuator does not receive the new optimal trajectory. In that case, the actuator implements the input corresponding to the last received trajectory. As the optimal trajectory is the solution of the LMPC optimization problem, it has been obtained using the predictions of the nominal model. Note that if the state is not available for a period longer than the prediction horizon, the actuator no longer can use the last received trajectory because the optimal trajectory of the LMPC optimization problem has a length equal to the prediction horizon, so after $N$ time steps without measurements, the input has to be kept constant at the last implemented value or set to a predefined value. In such a case, no guaranteed closed-loop stability or robustness properties can be obtained. This implementation procedure is summarized in the following algorithm:

1) If $s\left(t_{k}\right)=1$ then solve (22) and obtain $u_{k}^{*}(t)$, else $u_{k}^{*}(t)=$ $u_{k-1}^{*}(t)$

2) Apply $u(t)=u_{k}^{*}(t), t \in\left[t_{k}, t_{k+1}\right)$.

3) Obtain a new sample $(k=k+1)$ and go to 1 .

This strategy is a receding horizon strategy, which takes into account that data losses may occur. The actuator not only receives and implements a given input, but must also be able to store a future trajectory to implement it in case data losses occur. This implies that to improve the efficiency of a NCS, not only the control algorithms must be modified, but also the control actuator hardware that implements the control action. This scheme is motivated by the fact that when no feedback is available, a reasonable estimate of the future evolution of the system is given by the nominal trajectory. The proposed LMPC scheme, therefore modifies the standard implementation scheme of setting the actuator to zero or to the last computed input. Note that setting the input to zero is equivalent to assuming that the system has reached the origin, while setting the input to the last value is equivalent to assuming that the state remains constant throughout the time period on which data is lost. The idea of using the model to predict the evolution of the system when no feedback is possible has also been used in the context of sampled-data linear systems, see [24]-[29].

We now present two theorems that characterize the robustness properties of the proposed LMPC scheme if the process, controller, uncertainty and data loss parameters satisfy the conditions presented in the propositions $1-5$. The first theorem states that if no losses are present, the system converges to a neighborhood of the origin. The second theorem states that in the pres- 
ence of data losses that satisfy $N_{o} \leq N_{R}$ the closed-loop system state will not leave $\Omega_{\rho}$.

Theorem 1: Consider system (1) in closed-loop with the LPMC scheme (22) based on a controller $h(x)$ that satisfies (2). Let $\Delta, \rho, \rho_{s}, \rho^{*}, \epsilon_{s}, \epsilon_{w}$ and $\theta$ satisfy (7), (13) and (19). If there are no data losses $\left(s\left(t_{k}\right)=1, \forall k\right)$ and $x\left(t_{0}\right) \in \Omega_{\rho}$, then $x(t)$ is ultimately bounded in $\Omega_{\rho^{*}}$.

Proof: Taking into account Definition 1, for all $x\left(t_{k}\right) \in$ $\Omega_{\rho}$, the LMPC optimization problem is feasible because $u(t)=$ $h\left(\hat{x}\left(t_{k}\right)\right), t \in\left[t_{k}, t_{k+N_{R}}\right]$ satisfies the Lyapunov-based contractive constraint of (22). Following the same reasoning used in Proposition 3 to obtain (17), the following inequality is obtained (note that $x\left(t_{k}\right)$ is known because $s\left(t_{k}\right)=1$ ):

$V\left(x\left(t_{k+j}\right)\right) \leq V\left(\tilde{x}\left(t_{k+j}\right)\right)+\alpha_{4}\left(\alpha_{1}^{-1}(\rho)\right) \gamma_{e}(j \Delta)+\beta \gamma_{e}(j \Delta)^{2}$.

The constraints of (22) guarantee that $V(\tilde{x}(t)) \leq V(\hat{x}(t)), \forall t \in$ $\left[t_{k}, t_{k+N_{R}}\right]$ which implies that

$V\left(x\left(t_{k+j}\right)\right) \leq V\left(\hat{x}\left(t_{k+j}\right)\right)+\alpha_{4}\left(\alpha_{1}^{-1}(\rho)\right) \gamma_{e}(j \Delta)+\beta \gamma_{e}(j \Delta)^{2}$.

This inequality relates the actual trajectory $x(t)$ obtained in closed-loop with the LMPC controller, with the nominal sampled trajectory of the Lyapunov-based controller. We will use this inequality to bound the evolution of the Lyapunov function in the first time step in order to characterize the stability properties of the closed-loop system without data losses, i.e.,

$V\left(x\left(t_{k+1}\right)\right) \leq V\left(\hat{x}\left(t_{k+1}\right)\right)+\alpha_{4}\left(\alpha_{1}^{-1}(\rho)\right) \gamma_{e}(\Delta)+\beta \gamma_{e}(\Delta)^{2}$.

Since (7) holds, it follows from Proposition 2 that the nominal sampled trajectory $\hat{x}(t)$ satisfies

$$
V\left(\hat{x}\left(t_{k+1}\right)\right) \leq \max \left\{V\left(\hat{x}\left(t_{k}\right)\right)-\epsilon_{s}, \rho_{\min }\right\} .
$$

Using (25) into (24), and taking into account that (13) and (19) hold, the following inequality is obtained

$$
V\left(x\left(t_{k+1}\right)\right) \leq \max \left\{V\left(x\left(t_{k}\right)\right)-\epsilon_{w}, \rho^{*}\right\} .
$$

Using this inequality recursively the closed-loop trajectories of system (1) without data losses are characterized by means of the following expression

$$
\limsup _{k \rightarrow \infty} V\left(x\left(t_{k}\right)\right) \leq \rho^{*} .
$$

This proves that the closed-loop system is ultimately bounded in $\Omega_{\rho^{*}}$.

In the following theorem we present a result which guarantees that if the maximum time without measurements (i.e., $N_{o}$ ) is smaller than $N_{R}$, where $N_{R}$ is defined in Proposition 5, and the prediction horizon is chosen equal or greater than $N_{R}$, then $\Omega_{\rho}$ is an invariant set for system (1) controlled with the proposed LPMC scheme based on a controller that satisfies (2).

Theorem 2: Consider system (1) in closed-loop with the LPMC scheme (22) based on a controller $h(x)$ that satisfies (2). Let $\Delta, \rho, \rho_{s}, \epsilon_{s}, \epsilon_{w}, \theta$ satisfy (7), (13), (19) and $N_{R}$ is defined as in Proposition 5. If $x\left(t_{0}\right) \in \Omega_{\rho}$ and is known and $N_{o} \leq N_{R} \leq N$, then $x\left(t_{k}\right) \in \Omega_{\rho}$ for $t_{k}=t_{0}+k \Delta, k=0,1, \ldots$

Proof: We will prove in a recursive fashion that the theorem holds for the worst case trajectory with $N_{o} \leq N_{R}$. The worst case trajectory is given by $s\left(t_{k}\right)=1$ if $t_{k}=i N_{o}, i=$ $1,2, \ldots$ and $s\left(t_{k}\right)=0$ otherwise (that is, the system receives only one sample every $N_{o}$ sampling times).

We will first prove that if $x\left(t_{i N_{o}}\right) \in \Omega_{\rho}$ and is known (so the input at time $t_{i N_{o}}$ is taken on behalf of the real state), and the proposed implementation is applied, then $x\left(t_{(i+1) N_{o}}\right) \in \Omega_{\rho}$. At time $t_{(i+1) N_{o}}$ the actual state is available so $u^{*}(t)$ is evaluated and applied in $t \in\left[t_{i N_{o}}, t_{(i+1) N_{o}}\right]$. Note that the input is well defined for this period of time because $N \geq N_{o}$. Since $x\left(t_{i N_{o}}\right) \in \Omega_{\rho}$, we can apply Proposition 5 to prove that

$V\left(\hat{x}\left(t_{(i+1) N_{o}}\right)\right)+\alpha_{4}\left(\alpha_{1}^{-1}(\rho)\right) \gamma_{e}\left(N_{o} \Delta\right)+\beta \gamma_{e}\left(N_{o} \Delta\right)^{2} \leq \rho$.

Using (19) and (23) it follows that $x(t) \in \Omega_{\rho}$ for all $t \in$ $\left[t_{i N_{o}}, t_{(i+1) N_{o}}\right]$, and thus, $x\left(t_{(i+1) N_{o}}\right) \in \Omega_{\rho}$.

Taking into account that $x\left(t_{0}\right) \in \Omega_{\rho}$ and is known and using recursively the property that states that if $x\left(t_{i N_{o}}\right) \in \Omega_{\rho}$ and is known then $x\left(t_{(i+1) N_{o}}\right) \in \Omega_{\rho}$, it is proved that if $s\left(t_{k}\right)=1$ for $t_{k}=i N_{o}, i=1,2, \ldots$ and $s\left(t_{k}\right)=0$ otherwise, then $x\left(t_{k}\right) \in \Omega_{\rho}$ for $t_{k}=t_{0}+k \Delta, k=0,1, \ldots$.

Because the worst case trajectory is guaranteed to remain in $\Omega_{\rho}$ when the system is in closed-loop with the LMPC controller, then any given trajectory with $N_{o} \leq N_{R}$ is also guaranteed to remain in $\Omega_{\rho}$.

Remark 5: Theorem 2 is very important from an MPC point of view because if the maximum time without data losses is smaller than the maximum time that the system can operate in open-loop without leaving the stability region, the feasibility of the optimization problem for all times is guaranteed, since each time feedback is regained, the state is guaranteed to be inside the stability region, thereby yielding a feasible optimization problem.

Remark 6: In this work, no input or state constraints have been considered but the proposed approach can be extended to handle these issues. For input constraints, the Lyapunov-based controller has to be designed taking into account the limits on the inputs in an explicit way, such that a closed-loop stability region in which satisfaction of the constraints and asymptotic stability is guaranteed, is obtained. Example of such control laws are available in the literature for a for certain classes of nonlinear systems, see [45], [46] for results in this area. The LMPC optimization problem would also include the input and state constraints. Detailed development of theses results is outside the scope of the present work.

Remark 7: When there are data losses in the control system, standard MPC formulations do not provide guaranteed closedloop stability results because the stability properties of these controllers are proved based upon the assumption of flawless communications and in the presence of data losses the proofs are no longer valid. In order to guarantee closed-loop properties in the presence of data losses, the formulation of the optimization problem and the implementation procedure have to be modified accordingly to take into account data losses in an explicit way. 
TABLE I

PROCESS PARAMETERS

\begin{tabular}{|c|c|c|c|}
\hline$F$ & $4.998\left[\mathrm{~m}^{3} / \mathrm{h}\right]$ & $k_{10}$ & $3 * 10^{6}\left[h^{-1}\right]$ \\
\hline$V_{r}$ & $1\left[\mathrm{~m}^{3}\right]$ & $k_{20}$ & $3 * 10^{5}\left[h^{-1}\right]$ \\
\hline$R$ & $8.314[\mathrm{KJ} / \mathrm{kmol} \cdot \mathrm{K}]$ & $k_{30}$ & $3 * 10^{5}\left[h^{-1}\right]$ \\
\hline$T_{A 0}$ & $300[K]$ & $E_{1}$ & $5 * 10^{4}[\mathrm{KJ} / \mathrm{kmol}]$ \\
\hline$C_{A 0}$ & $4\left[\mathrm{kmol} / \mathrm{m}^{3}\right]$ & $E_{2}$ & $7.53 * 10^{4}[\mathrm{KJ} / \mathrm{kmol}]$ \\
\hline$\Delta H_{1}$ & $-5.0^{*} 10^{4}[\mathrm{KJ} / \mathrm{kmol}]$ & $E_{3}$ & $7.53 * 10^{4}[\mathrm{KJ} / \mathrm{kmol}]$ \\
\hline$\Delta H_{2}$ & $-5.2 * 10^{4}[\mathrm{KJ} / \mathrm{kmol}]$ & $\sigma$ & $1000\left[\mathrm{~kg} / \mathrm{m}^{3}\right]$ \\
\hline$\Delta H_{3}$ & $-5.4 * 10^{4}[\mathrm{KJ} / \mathrm{kmol}]$ & $c_{p}$ & $0.231[\mathrm{KJ} / \mathrm{kg} \cdot \mathrm{K}]$ \\
\hline
\end{tabular}

\section{Application to A CHEMICAL REACTOR}

Consider a well mixed, non-isothermal continuous stirred tank reactor where three parallel irreversible elementary exothermic reactions take place of the form $A \rightarrow B, A \rightarrow C$ and $A \rightarrow D$. $\mathrm{B}$ is the desired product and $\mathrm{C}$ and $\mathrm{D}$ are byproducts. The feed to the reactor consists of pure A at flow rate $F$, temperature $T_{A 0}$ and molar concentration $C_{A 0}+\Delta C_{A 0}$ where $\Delta C_{A 0}$ is an unknown time-varying uncertainty. Due to the non-isothermal nature of the reactor, a jacket is used to remove/provide heat to the reactor. Using first principles and standard modeling assumptions the following mathematical model of the process is obtained

$$
\begin{aligned}
\frac{d T}{d t} & =\frac{F}{V_{r}}\left(T_{A 0}-T\right)-\sum_{i=1}^{3} \frac{\Delta H_{i}}{\sigma c_{p}} k_{i 0} e^{\frac{-E_{i}}{R T}} C_{A}+\frac{Q}{\sigma c_{p} V_{r}} \\
\frac{d C_{A}}{d t} & =\frac{F}{V_{r}}\left(C_{A 0}+\Delta C_{A 0}-C_{A}\right)+\sum_{i=1}^{3} k_{i 0} e^{\frac{-E_{i}}{R T}} C_{A}
\end{aligned}
$$

where $C_{A}$ denotes the concentration of the reactant $A, T$ denotes the temperature of the reactor, $Q$ denotes the rate of heat input/removal, $V_{r}$ denotes the volume of the reactor, $\Delta H_{i}, k_{i 0}, E_{i}, i=1,2,3$ denote the enthalpies, pre-exponential constants and activation energies of the three reactions, respectively, $c_{p}$ and $\sigma$ denote the heat capacity and the density of the fluid in the reactor. The values of the process parameters are shown in Table I.

System (26) has three steady-states (two locally asymptotically stable and one unstable). The control objective is to stabilize the system at the open-loop unstable steady state $T_{s}=$ $388 K, C_{A s}=3.59 \mathrm{~mol} / \mathrm{l}$. The manipulated input is the rate of heat input $Q$. We consider a time-varying uncertainty in the concentration of the inflow $\left|\Delta C_{A 0}\right| \leq 0.5 \mathrm{kmol} / \mathrm{m}^{3}$.

To demonstrate the theoretical results, we are going to apply three different controllers: A Lyapunov-based controller that satisfies (2), the original LMPC controller (5), and the proposed LMPC controller (22). We first design a Lyapunov-based controller using the method presented in [47]. This controller is used to design both LMPC controllers. System (26) belongs to the following class of non-linear systems

$$
\dot{x}(t)=f(x(t))+g(x(t)) u(t)+w(x(t)) \theta(t)
$$

where $x^{T}=\left[T C_{A}\right]$ is the state, $u=Q$ is the input and $\theta=\Delta C_{A 0}$ is a time varying bounded disturbance. Consider the control Lyapunov function $V(x)=x^{T} P x$ with

$$
P=\left[\begin{array}{cc}
1 & 0 \\
0 & 10^{4}
\end{array}\right] \text {. }
$$

The values of the weights have been chosen to account for the different range of numerical values for each state. The following feedback law [47] asymptotically stabilizes the open-loop unstable steady-state of the nominal system (i.e., $\theta(t)=0$ ) and is of the form (2):

$$
h(x)= \begin{cases}-\frac{L_{f} V+\sqrt{L_{f} V^{2}+L g V^{4}}}{L_{g} V} & \text { if } L_{g} V \neq 0 \\ 0 & \text { if } L_{g} V=0\end{cases}
$$

where $L_{f} V$ and $L_{g} V$ denote the Lie derivatives of the scalar function $V$ with respect to the vectors fields $f$ and $g$, respectively. This controller will be used to design the LMPC controller. The stability region $\Omega_{\rho}$ is defined as $V(x) \leq 1000$, i.e., $\rho=1000$.

The main source of asynchronous behavior in the closed-loop system of this chemical process example is the presence of asynchronous measurement sampling which is meaningful from a practical point of view given the type and size of the LMPC optimization problem and the amount of data involved in the transmission of the input trajectory from the controller to the actuator. We assume that the sampling of the concentration of product A occurs every three to ten minutes. Although the temperature sampling is much faster, a measurement of the full process state vector $\left(C_{A}, T\right)$ is sent to the controller only when the concentration measurement is available. We model the asynchronous measurement sampling of the closed-loop system with a sampled-data system with a sampling time $\Delta=0.05 h$ subject to data losses. For this sampling time, the closed-loop system with $u=h(x)$ is practically stable and the performance in terms of the evolution of the process states is similar to the closed-loop system with continuous measurements. We computed the maximum time such that the system remains in $\Omega_{\rho}$ when controlled in open-loop (i.e., no measurement of the full process state vector are available to the controller apart from the initial state) with the nominal sampled input trajectory to be $5 \Delta$ (i.e., $N_{R}=5$ ). This value is estimated using data from simulations. As expected, the inequalities obtained in the main results of this paper are too conservative to realistically estimate $\Delta$ and $N_{R}$.

We implement the LMPC controller presented in the previous section using a sample time of $\Delta=0.05 h$ and a prediction horizon $N=N_{R}=5$. The cost function is defined by the weight matrices $Q_{c}=P$ and $R_{c}=10^{-6}$. The values of the weights have been tuned in such a way that the values of the control inputs are comparable to the ones computed by the Lyapunov-based controller (i.e., same order of magnitude of the input signal and convergence time of the closed-loop system when no uncertainty or losses are taken into account).

We will first compare the proposed LMPC scheme with the original LMPC scheme of [33]-[35]. In this scheme no data losses were taken into account. This scheme is based on an optimization problem of the form (5) and will react to data loss with the same approach as the proposed LMPC scheme, that is, sending to the actuator the whole optimal input trajectory, so in case data losses occur, the input is updated as in the modified receding horizon scheme proposed in Section IV. The same weights, sampling time and prediction horizon are used.

In Fig. 2 the trajectories of both controllers are shown assuming no data is lost, that is, the state $x\left(t_{k}\right)$ is available every 

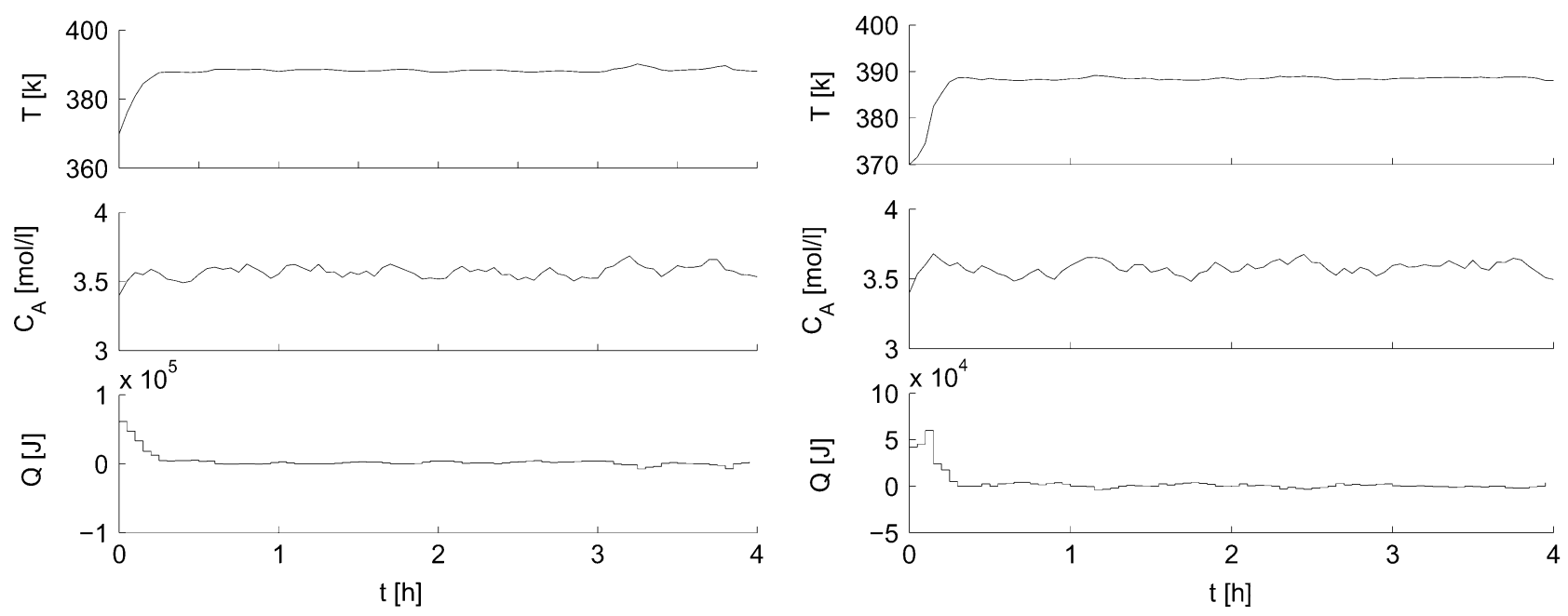

(a)

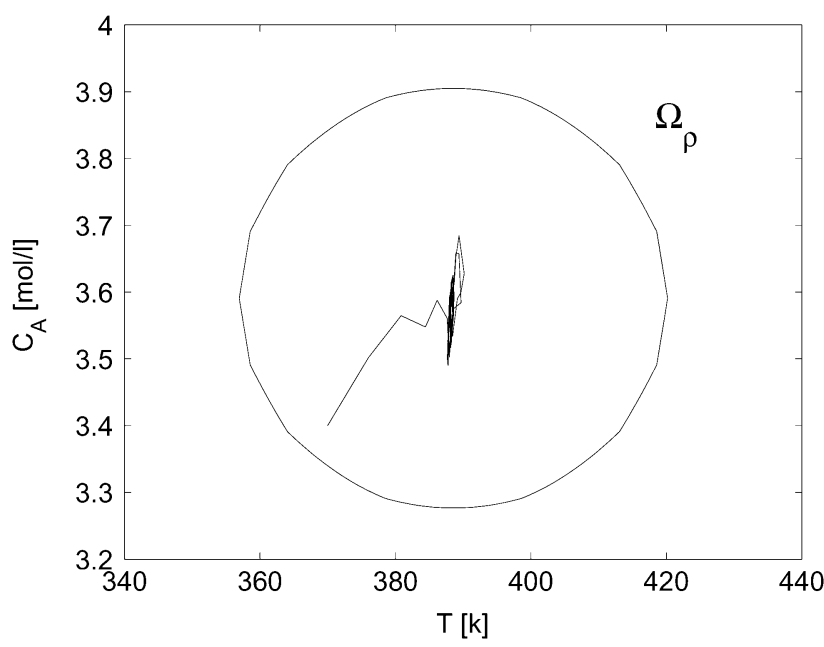

(c)

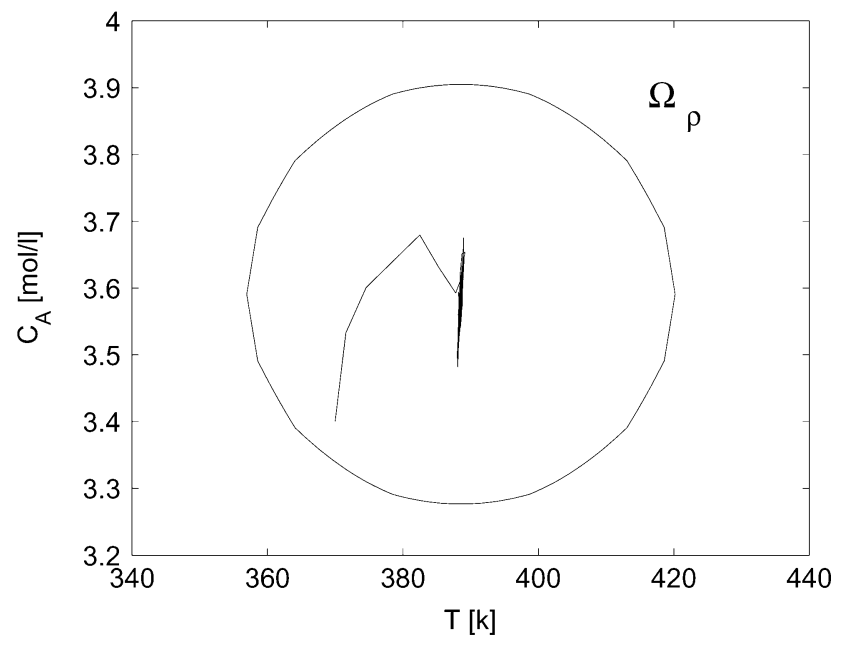

(d)

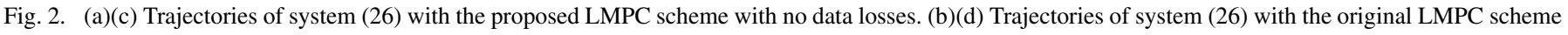
with no data losses.

sampling time. It can be seen that both closed-loop systems are practically stable. Note that regarding optimality, for a given state, the original LMPC optimization problem (not necessarily the closed-loop trajectory) yields a lower cost than the proposed optimization problem, because the constraints that define the original LMPC controller are less restrictive (i.e., the contractive constraint must hold only in the first time step whereas in the proposed LMPC scheme it must hold along the whole prediction horizon). The difference between the optimal cost of the original LMPC and the proposed LMPC for a particular initial state depends on whether the contractive constraint (25d) is active or not (i.e., satisfied with equality sign) at the solution. In general, the constraint is more restrictive for states near the boundary of the stability region. For $x=[370 ; 3.4]$, the original LMPC yields $J^{*}(x)=7138.8$ and the proposed controller yields $J^{*}(x)=8238.6$. For $x=[384 ; 3.55]$, the original LMPC yields $J^{*}(x)=457.9$, while the proposed controller yields $J^{*}(x)=472.5$.

When data losses occur, the proposed LMPC scheme is more robust. The stability region is invariant for the closed-loop system if $N_{o} \leq N$ (recall that we set the prediction horizon equal to the maximum time defined in Proposition 5). That is not the case with the LMPC scheme of (5). In Fig. 3 the trajectories of the closed-loop system under both controllers are shown in the presence of data losses with $N_{o}=5$, and a signal $s\left(t_{k}\right)$ evaluated as $s\left(t_{k}\right)=1$ if $t_{k}=i N_{o}, i=1,2, \ldots$ and $s\left(t_{k}\right)=0$ otherwise (that is, the system receives only one measurement of the actual state every 5 samples). These trajectories account for the worst-case effect of the data losses. The trajectories are shown in the state space along with the closed-loop stability region $\Omega_{\rho}$. It can be seen that the original LMPC controller trajectory leaves the stability region, while the proposed LMPC scheme remains inside. When data losses are taken into account, in order to inherit the stability properties of the Lyapunov-based controller of (27), the constraints must be modified to take into account data losses as in the proposed LMPC controller (22).

We now compare the LMPC with the Lyapunov-based controller (27) applied in a sample-and-hold fashion following a "last available control" strategy, i.e., when data is lost, the actuator keeps implementing the last received input value (note that, through extensive simulations, we have found that in this 

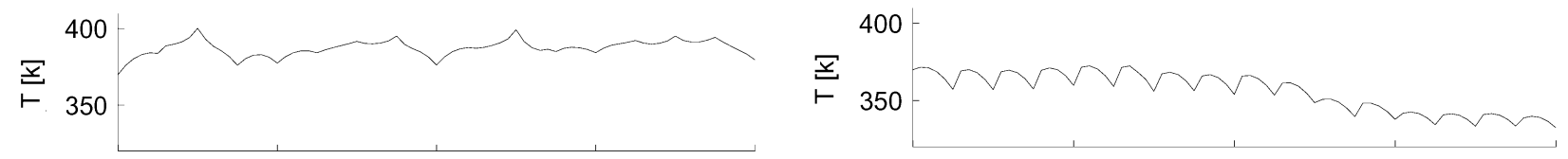

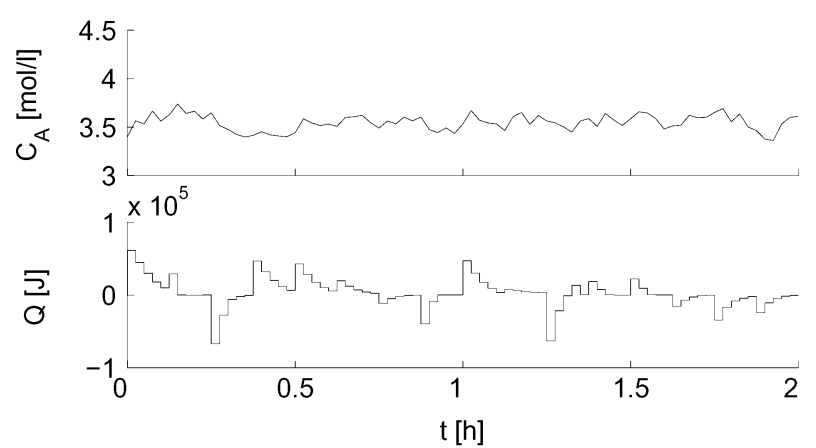

(a)

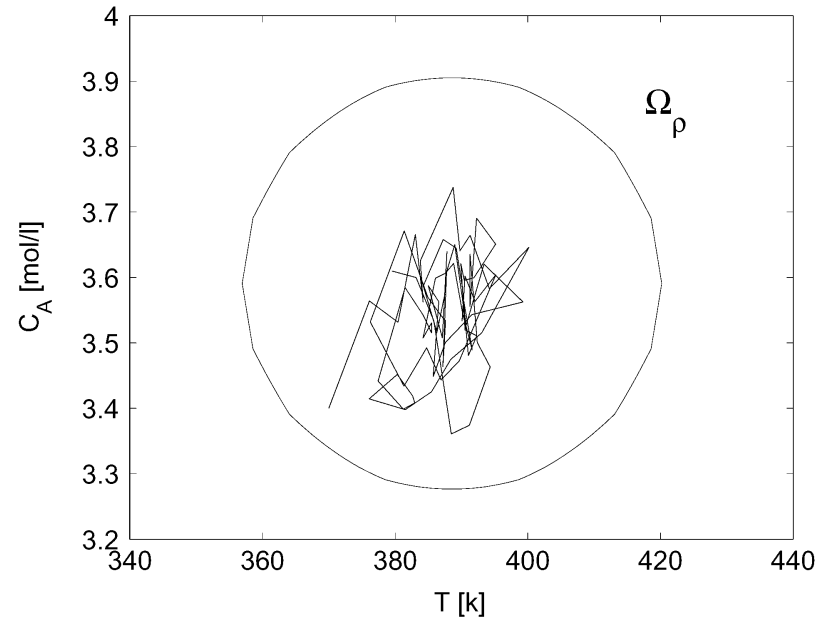

(c)

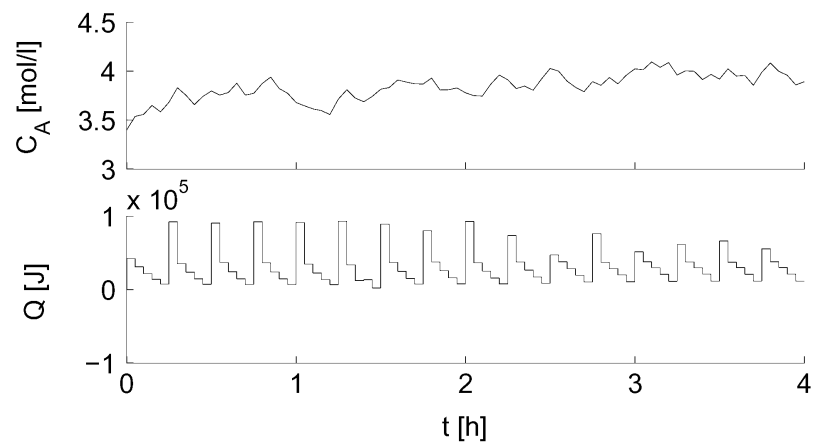

(b)

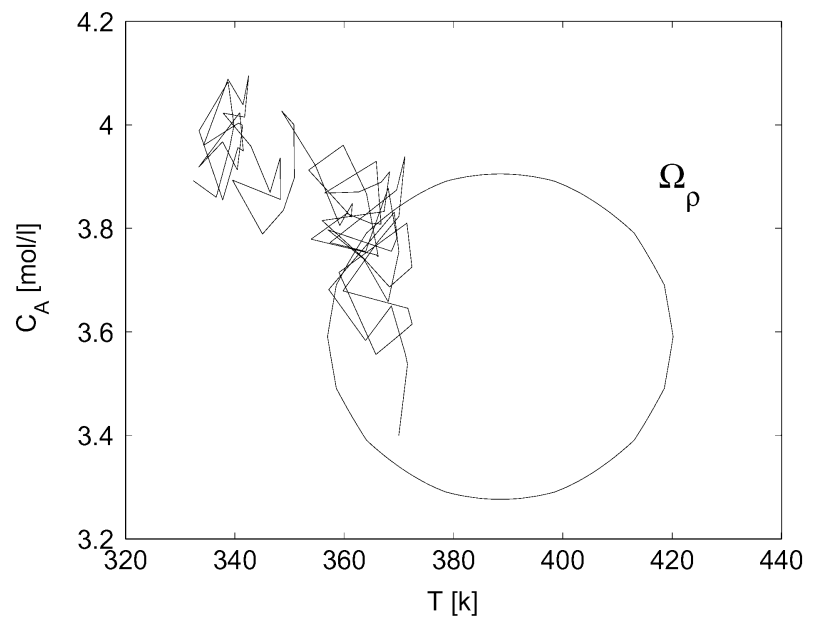

(d)

Fig. 3. (a)(c) Worst case trajectories of system (26) with the proposed LMPC scheme with $N_{o}=5$. (b)(d) Worst case trajectories of system (26) with the original LMPC scheme with $N_{0}=5$.

particular example, the strategy of setting the input to zero when data losses occur, yields worse results than the strategy of implementing the last available input). In Fig. 4 the worst case trajectories with $N_{o}=2$ for both controllers are shown (note that this loss rate is lower than the one that can be handled by the proposed LMPC controller). It can be seen that, due to the instability of the open-loop steady state, for this small amount of losses, the Lyapunov-based controller is not able to stabilize the system. This is due to the fact that this control scheme does not update the control actuator output using the model, as the proposed LMPC scheme does.

We have also carried out another set of simulations to demonstrate that although the LMPC scheme inherits the same stability and robustness properties of the Lyapunov-based controller that it employs, it does outperform the Lyapunov-based controller (27) from a performance index point of view. Table II shows the total cost computed for 10 different closed-loop simulations under the LMPC and the Lyapunov-based controller implemented in a sample-and-hold fashion, using the nominal model to predict the evolution of the system when data is lost. To carry out this comparison, we compute the total cost of each simulation based on the performance index of the LMPC which has the form

$$
\sum_{i=0}^{M} x\left(t_{i}\right)^{T} Q_{c} x\left(t_{i}\right)+u\left(t_{i}\right)^{T} R_{c} u\left(t_{i}\right)
$$

where $t_{0}$ is the initial time of the simulations and $t_{M}=4 \mathrm{~h}$ is the end of the simulation. The sampling time is $\Delta=0.05 \mathrm{~h}$. Each sample has a $20 \%$ probability of being lost, that is $P\left(s\left(t_{k}\right)=\right.$ $0)=0.2$. For each pair of simulations (one for each controller) a different initial state inside the stability region, a different random uncertainty trajectory and a different data losses realization is chosen. As it can be seen in Table II, the total cost under the proposed LMPC controller is lower than the corresponding total cost under the Lyapunov-based controller. This demonstrates that in this example, the LMPC scheme shares the same robustness and stability properties and is more optimal than the Lyapunov-based controller, which is not designed taking into account any optimality considerations.

The simulations have been done in Matlab using fmincon and a Runge-Kutta solver with a fixed integration time of 

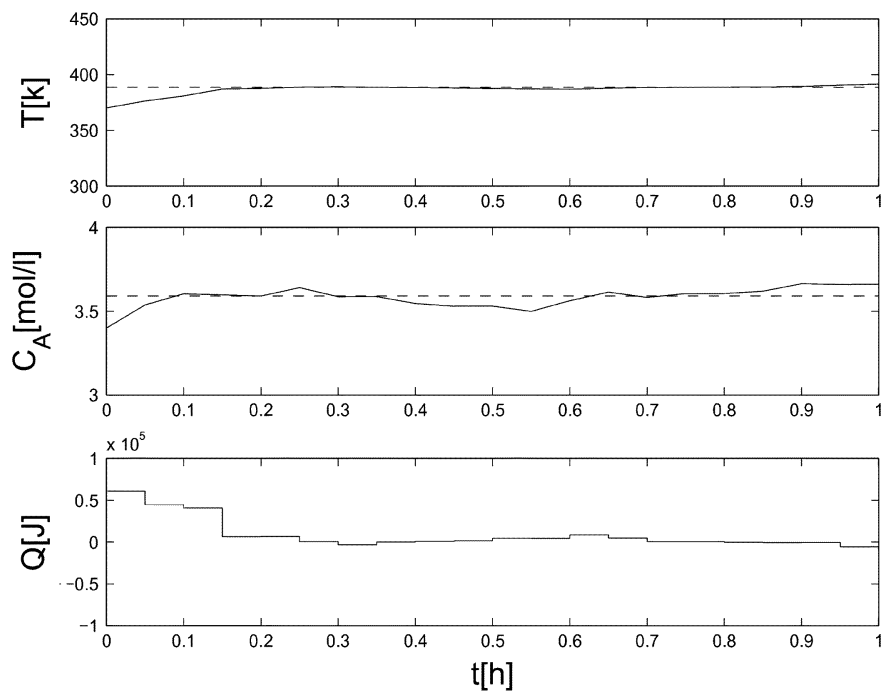

(a)
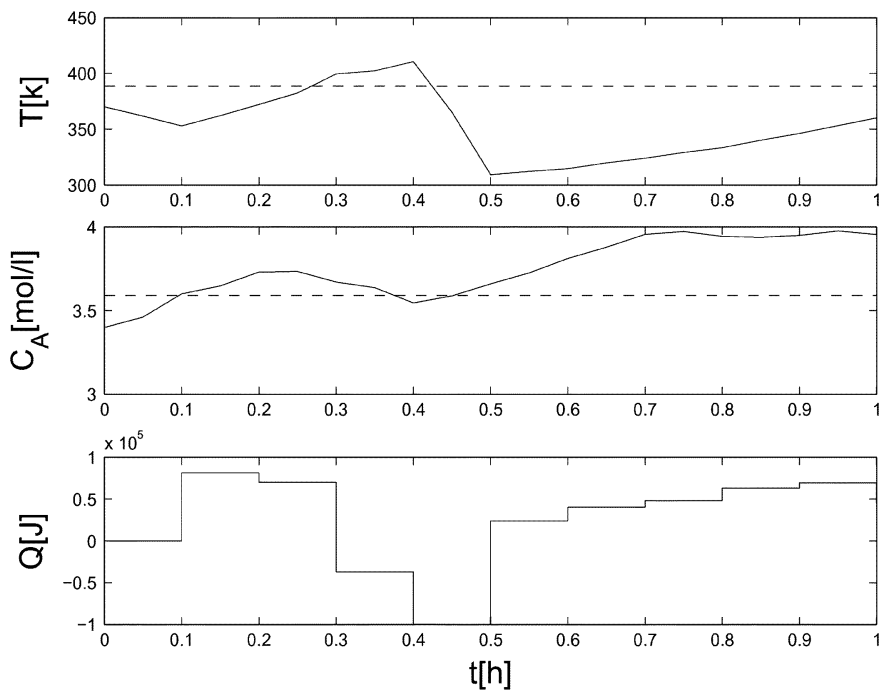

(b)

Fig. 4. Worst case trajectories of system (26) with $N_{o}=2$ in closed-loop with (a) the proposed LMPC scheme; (b) the Lyapunov-based controller.

TABLE II

Performance Costs Along the Closed-Loop Trajectories

\begin{tabular}{|c|cc|}
\hline sim. & Lyapunov-based controller & LMPC \\
\hline 1 & $0.1262 * 10^{12}$ & $0.0396 * 10^{12}$ \\
2 & $0.3081 * 10^{12}$ & $0.2723 * 10^{12}$ \\
3 & $0.0561 * 10^{12}$ & $0.0076 * 10^{12}$ \\
4 & $0.9622 * 10^{11}$ & $0.2884 * 10^{11}$ \\
5 & $3.8176 * 10^{11}$ & $1.3052 * 10^{11}$ \\
6 & $0.9078 * 10^{11}$ & $0.0950 * 10^{11}$ \\
7 & $0.4531 * 10^{12}$ & $0.2678 * 10^{12}$ \\
8 & $0.6752 * 10^{11}$ & $0.5689 * 10^{11}$ \\
9 & $1.0561 * 10^{11}$ & $0.6776 * 10^{11}$ \\
10 & $0.5332 * 10^{12}$ & $0.3459 * 10^{12}$ \\
\hline
\end{tabular}

0.001h. To simulate the time-varying uncertainty, a different random value $\theta(t)$ has been applied at each integration step. This random value satisfies $|\theta(t)| \leq 0.5 \mathrm{kmol} / \mathrm{m}^{3}$ and is taken from a uniform distribution using Matlab's function rand.

\section{CONCLUSION}

In this work, a LMPC controller was proposed for control of a broad class of nonlinear uncertain systems subject to data losses.
The main idea is that in order to provide guaranteed stability results in the presence of data losses, the constraints that define the LMPC optimization problem as well as the implementation procedure have to be modified to account for data losses. This fact also holds for other MPC controllers. The proposed LMPC controller allows for an explicit characterization of the stability region, guarantees practical stability in the absence of data losses, and guarantees that the stability region is invariant if the maximum time without measurements is shorter than a given constant that depends on the parameters of the system and the controller used to design the LMPC. The proposed control method was demonstrated though application to a chemical process example.

\section{REFERENCES}

[1] W. Zhang, M. S. Branicky, and S. M. Phillips, "Stability of networked control systems," IEEE Control Syst. Mag., vol. 21, pp. 84-89, 2001.

[2] T. C. Yang, "Networked control systems: A brief survey," Proc. Inst. Elect. Eng., vol. 153, pp. 403-412, 2006.

[3] P. Neumann, "Communication in industrial automation-what is going on?," Control Eng. Practice, vol. 15, pp. 1332-1347, 2007.

[4] G. T. Nguyen, R. H. Katz, B. Noble, and M. Satyanarayananm, "A tracebased approach for modeling wireless channel behavior," in Proc. Winter Simul. Conf., 1996, pp. 597-604.

[5] N. J. Ploplys, P. A. Kawka, and A. G. Alleyne, "Closed-loop control over wireless networks-Developing a novel timing scheme for realtime control systems," IEEE Control Syst. Mag., vol. 24, pp. 52-71, 2004.

[6] P. A. Kawka and A. G. Alleyne, "Stability and feedback control of wireless networked systems," in Proc. Amer. Control Conf., 2005, pp. 2955-2959.

[7] M. Tabbara, D. Nesic, and A. R. Teel, "Stability of wireless and wireline control systems," IEEE Trans. Automat. Control, vol. 52, no. 9, pp. 1615-1630, Sep. 2007.

[8] I. F. Akyildiz, W. Su, Y. Sankarasubramaniam, and E. Cayirci, "Wireless sensor networks: A survey," Comput. Networks-Int. J. Comput. Telecommun. Networking, vol. 38, pp. 393-422, 2002.

[9] C. Y. Chong and S. P. Kumar, "Sensor networks: Evolution, opportunities, and challenges," Proc. IEEE, vol. 91, no. 8, pp. 1247-1256, Aug. 2003.

[10] W. Caripe, G. Cybenko, K. Moizumi, and R. Gray, "Network awareness and mobile agent systems," IEEE Commun. Mag., vol. 36, no. 7, pp. 44-49, Jul. 1998.

[11] P. Wilke and T. Braunl, "Flexible wireless communication network for mobile robot agents," Ind. Robot-Int. J., vol. 28, pp. 220-232, 2001.

[12] P. Mhaskar, A. Gani, C. McFall, P. D. Christofides, and J. F. Davis, "Fault-tolerant control of nonlinear process systems subject to sensor data losses," AIChE J., vol. 53, pp. 654-668, 2007.

[13] W. Zhang and M. S. Branicky, "Stability of networked control systems with time-varying transmission period," in Proc. Allerton Conf., 2001, [CD ROM].

[14] A. Hassibi, S. P. Boyd, and J. P. How, "Control of asynchronous dynamical systems with rate constraints on events," in Proc. IEEE Conf. Decision Control, 1999, pp. 1345-1351.

[15] H. Lin and P. J. Antsaklis, "Stability and persistent disturbance attenuation properties for a class of networked control systems: Switched system approach," Int. J. Control, vol. 78, pp. 1447-1458, 2005.

[16] O. C. Imer, S. Yüksel, and T. Başar, "Optimal control of LTI systems over unreliable communications links," Automatica, vol. 42, pp. 1429-1439, 2006.

[17] C. N. Hadjicostis and R. Touri, "Feedback control utilizing packet dropping networks links," in Proc. IEEE Conf. Decision Control, Las Vegas, NV, 2001, pp. 1205-1210.

[18] B. Azimi-Sadjadi, "Stability of networked control systems in the presence of packet losses," in Proc. IEEE Conf. Decision Control, Maui, HI, 2003, pp. 676-681.

[19] N. Elia and J. N. Eisenbeis, "Limitations of linear remote control over packet drop networks," in Proc. IEEE Conf. Decision Control, Nassau, Bahamas, 2004, pp. 5152-5157.

[20] G. Walsh, O. Beldiman, and L. Bushnell, "Asymptotic behavior of nonlinear networked control systems," IEEE Trans. Automat. Control, vol. 46, no. 7, pp. 1093-1097, Jul. 2001. 
[21] G. Walsh, H. Ye, and L. Bushnell, "Stability analysis of networked control systems," IEEE Trans. Control Syst. Technol., vol. 10, no. 5, pp. 438-446, Sep. 2002.

[22] D. Nešić and A. R. Teel, "Input-to-state stability of networked control systems," Automatica, vol. 40, pp. 2121-2128, 2004.

[23] D. Nešić and A. R. Teel, "Input-output stability properties of networked control systems," IEEE Trans. Automat. Control, vol. 49, no. 10, pp. 1650-1667, Oct. 2004.

[24] L. A. Montestruque and P. J. Antsaklis, "On the model-based control of networked systems," Automatica, vol. 39, pp. 1837-1843, 2003.

[25] L. A. Montestruque and P. J. Antsaklis, "Stability of model-based networked control systems with time-varying transmission times," IEEE Trans. Automat. Control, vol. 49, no. 9, pp. 1562-1572, Sep. 2004.

[26] P. Naghshtabrizi and J. Hespanha, "Designing observer-type controllers for network control systems," in Proc. IEEE Conf. Decision Control, 2005, pp. 848-853.

[27] P. Naghshtabrizi and J. Hespanha, "Anticipative and non-anticipative controller design for network control systems, network embedded sensing and control," Netw. Embedded Sens. Control Lecture Notes Control Inform. Sci., vol. 331, pp. 203-218, 2006.

[28] W. J. Kim, K. Ji, and A. Ambike, "Networked real-time control strategy dealing with stochastic time delays and packet losses," ASME J. Dynam. Syst., Meas. Control, vol. 128, pp. 681-685, 2006.

[29] W. J. Kim, K. Ji, and A. Srivastava, "Network-based control with realtime prediction of delayed/lost sensor data," IEEE Trans. Control Syst. Technol., vol. 14, no. 1, pp. 182-185, Jan. 2006.

[30] E. F. Camacho and C. Bordons, Model Predictive Control, 2nd ed. Berlin, Germany: Springer-Verlag, 2004.

[31] J. M. Maciejowski, Predictive Control with Constraints. Englewood Cliffs, NJ: Prentice Hall, 2002.

[32] D. Q. Mayne, J. B. Rawlings, C. V. Rao, and P. O. M. Scokaert, "Constrained model predictive control: Stability and optimality," Automatica, vol. 36, pp. 789-814, 2000.

[33] P. Mhaskar, N. H. El-Farra, and P. D. Christofides, "Predictive control of switched nonlinear systems with scheduled mode transitions," IEEE Trans. Automatic Control, vol. 50, no. 11, pp. 1670-1680, Nov. 2005.

[34] P. Mhaskar, N. H. El-Farra, and P. D. Christofides, "Stabilization of nonlinear systems with state and control constraints using Lyapunovbased predictive control," Syst. Control Lett., vol. 55, pp. 650-659, 2006.

[35] P. Mhaskar, A. Gani, and P. D. Christofides, "Fault-tolerant control of nonlinear processes: Performance-based reconfiguration and robustness," Int. J. Robust Nonlin. Control, vol. 16, pp. 91-111, 2006.

[36] J. A. Primbs, V. Nevistic, and J. C. Doyle, "A receding horizon generalization of pointwise min-norm controllers," IEEE Trans. Automat. Control, vol. 45, no. 5, pp. 898-909, May 2000.

[37] S. L. D. Kothare and M. Morari, "Contractive model predictive control for constrained nonlinear systems," IEEE Trans. Automat. Control, vol. 45, no. 6, pp. 1053-1071, Jun. 2000.

[38] H. S. Witsenhausen, "A min-max control problem for sampled linear systems," IEEE Trans. Automat. Control, vol. AC-13, no. 1, pp. 5-21, Jan. 1968.

[39] P. J. Campo and M. Morari, "Robust model predictive control," in Proc. Amer. Control Conf., 1987, vol. 2, pp. 1021-1026.

[40] P. O. M. Scokaert and D. Q. Mayne, "Min-max feedback model predictive control for constrained linear systems," IEEE Trans. Automat. Control, vol. 43, no. 8, pp. 1136-1142, Aug. 1998.

[41] T. Alamo, D. Muñoz de la Peñia, D. Limon, and E. F. Camacho, "Constrained minmax predictive control: Modifications of the objective function leading to polynomial complexity," IEEE Trans. Automat. Control, vol. 50, no. 5, pp. 710-714, May 2005.

[42] D. Muñoz de la Peña, T. Alamo, A. Bemporad, and E. F. Camacho, "A decomposition algorithm for feedback min-max model predictive control," IEEE Trans. Automat. Control, vol. 51, no. 10, pp. 1688-1692, Oct. 2006.

[43] J. L. Massera, "Contributions to stability theory," Annals Math., vol. 64, pp. 182-206, 1956.

[44] H. K. Khalil, Nonlinear Systems, 3rd ed. Englewood Cliffs, NJ: Prentice Hall, 2002.

[45] P. Kokotovic and M. Arcak, "Constructive nonlinear control: A historical perspective," Automatica, vol. 37, pp. 637-662, 2001.
[46] P. D. Christofides and N. H. El-Farra, Control of Nonlinear and Hybrid Process Systems: Designs for Uncertainty, Constraints and Time-Delays. Berlin, Germany: Springer-Verlag, 2005.

[47] E. Sontag, "A 'universal' construction of arstein's theorem on nonlinear stabilization," Syst. Control Lett., vol. 13, pp. 117-123, 1989

[48] F. Clarke, Y. Ledyaev, and E. Sontag, "Asymtotic controllability implies feedback stabilization," IEEE Trans. Automat. Control, vol. 42, no. 10, pp. 1394-1407, Oct. 1997.

[49] D. Nešić, A. Teel, and P. Kokotovic, "Sufficient conditions for stabilization of sampled-data nonlinear systems via discrete time approximations," Syst. Control Lett., vol. 38, pp. 259-270, 1999.

[50] D. Liberzon, "On stabilization of linear systems with limited information," IEEE Trans. Automat. Control, vol. 48, no. 2, pp. 304-307, Feb. 2003.

[51] P. Tabuada and X. Wang, "Preliminary results on state-trigered scheduling of stabilizing control tasks," in Proc. IEEE Conf. Decision Control, 2006, pp. 282-287.

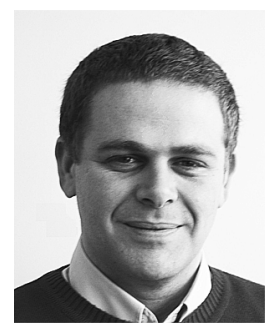

David Muñoz de la Peña received the M.S. degree in telecommunication engineering and the $\mathrm{Ph} . \mathrm{D}$. degree in control engineering from the University of Seville, Seville, Spain, in 2001 and 2005, respectively.

From July 2003 to June 2004, he was a visiting graduate student with the Department of Information Engineering, University of Siena, Siena, Italy. From March 2006 to April 2007, he was a Postdoctoral Researcher with the Department of Chemical and Biomolecular Engineering, University of California, Los Angeles. Since May 2007, he has been with the Department of System Engineering and Automation, University of Seville, where he is currently an Associate Professor. His main research interests are model predictive control, nonlinear systems, and optimization.

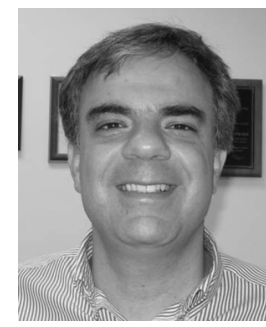

Panagiotis D. Christofides was born in Athens, Greece, in 1970. He received the Diploma in chemical engineering degree from the University of Patras, Patras, Greece, in 1992, and dual M.S. degrees in electrical engineering and mathematics and the Ph.D. degree in chemical engineering from the University of Minnesota, Minneapolis, in 1995 and 1996, respectively.

Since July 1996, he has been with the University of California, Los Angeles, where he is currently a Professor in the Department of Chemical and Biomolecular Engineering and the Department of Electrical Engineering. His theoretical research interests include nonlinear control, singular perturbations, and analysis and control of distributed parameter systems, multiscale systems and hybrid systems, with applications to advanced materials processing, particulate processes, biological systems, water systems and fluid flows. His research work has resulted in a large number of articles in leading scientific journals and conference proceedings and three books entitled Nonlinear and Robust Control of PDE Systems: Methods and Applications to Transport-Reaction Processes (Basil, Switzerland: Birkhäuser, 2001), Model-Based Control of Particulate Processes (Norwell, MA: Kluwer Academic, 2002) and Control of Nonlinear and Hybrid Process Systems: Designs for Uncertainty, Constraints and Time-Delays (Berlin, Germany: Springer, 2005).

Dr. Christofides received the Teaching Award from the AIChE Student Chapter of UCLA in 1997, the Research Initiation Award from the ACS-Petroleum Research Fund in 1998, the CAREER award from the National Science Foundation in 1998, the Ted Peterson Student Paper Award from the Computing and Systems Technology Division of AIChE in 1999, the Young Investigator Award from the Office of Naval Research in 2001, and the O. Hugo Schuck Best Paper Award in 2000 and 2004, and the Donald P. Eckman Award in 2004 from the American Automatic Control Council. He was a Plenary Speaker in the 2005 American Control Conference. 